\title{
Investigating the fit between phonological feature systems and brain responses to speech using EEG
}

\author{
Daniel R. McCloy and Adrian K. C. LeE \\ University of Washington, Institute for Learning and Brain Sciences, 1715 NE \\ Columbia Rd., Seattle, WA 98195-7988
}

\begin{abstract}
This paper describes a technique to assess the correspondence between patterns of similarity in the brain's response to speech sounds and the patterns of similarity encoded in phonological feature systems, by quantifying the recoverability of phonological features from the neural data using supervised learning. The technique is applied to EEG recordings collected during passive listening to consonant-vowel syllables. Three published phonological feature systems are compared, and are shown to differ in their ability to recover certain speech sound contrasts from the neural data. For the phonological feature system that best reflects patterns of similarity in the neural data, a leave-one-out analysis indicates some consistency across subjects in which features have greatest impact on the fit, but considerable across-subject heterogeneity remains in the rank ordering of features in this regard.
\end{abstract}

KEYWORDS: phonological features; EEG

\section{INTRODUCTION}

Phonemes are abstract representations of speech sounds that represent all and only the contrastive relationships between sounds (i.e., the set of sounds different enough to change the identity of a word if one sound were substituted for another; Jones, 1957). Within any given language, the set of phonemes is widely held to be structured in terms of phonological distinctive features (hereafter "phonological features") - properties common to some subset of the phoneme inventory. For example, in some phonological feature systems, all phonemes characterised by sounds made with complete occlusion of the oral tract share the feature "non-continuant." There are a number of competing hypotheses regarding the particular details of what the features are, and whether there is a single universal feature system or a separate feature system for each language.

Phonological features have been called the most important advance of linguistic theory of the 20th century, for their descriptive power in capturing sound patterns, and for their potential to capture the structure of phonological knowledge as represented in the brain (Mielke \& Hume, 2006). Few linguists would dispute the first of these claims; as every first-year phonology student learns, descriptions of how a phoneme is realised differently in different contexts can be readily generalised to other phonemes undergoing similar pronunciation changes if the change is expressed in terms of phonological features rather than individual sounds. To give a common

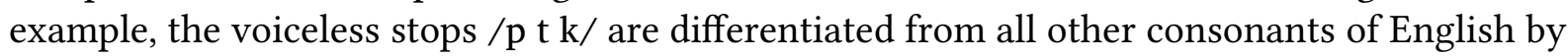
being the "-voiced, -continuant, -delayedRelease" sounds (in the feature system of Hayes, 2009). 
This allows contextual sound changes that apply only to the voiceless stops (such as aspiration before stressed vowels) to be succinctly described. Phonological features work equally well for describing changes that occur over long periods of time as languages evolve (e.g., Grimm's law: Bromberger \& Halle, 1989).

In contrast, the promise of phonological features as a model of speech sound representation or processing in the human brain is far from being conclusively established. There is growing evidence that the superior temporal gyrus represents acoustic-phonetic features - the spectrotemporal correlates of articulatory postures and gestures - such as voice onset time, vowel formant frequency, or other spectrotemporal properties of speech (Leonard, Bouchard, Tang, \& Chang, 2015; Mesgarani, Cheung, Johnson, \& Chang, 2014). Evidence also points to representations of phoneme or syllable identity in adjacent temporal areas such as posterior superior temporal sulcus (Vaden, Muftuler, \& Hickok, 2010) and the sylvian parieto-temporal region (Hickok, Okada, \& Serences, 2009), as well as frontal areas such as left inferior frontal sulcus (Markiewicz \& Bohland, 2016) and left precentral gyrus and sulcus (Evans \& Davis, 2015). However, studies that have looked for evidence of phonological feature representations in the brain have often used stimuli that don't distinguish whether the neural representation reflects a truly abstract phonological category or merely spectrotemporal properties shared among the stimuli (Arsenault \& Buchsbaum, 2015), and in one study that took pains to rule out the possibility of spectrotemporal similarity, no evidence for the representation of phonological features was found (Evans \& Davis, 2015).

Moreover, in most studies of how speech sounds are represented in the brain, the choice of which features to investigate (and which stimuli to use in studying them) rarely incorporates information about which sounds pattern together in speech, and thus rarely provides evidence for or against any particular phonological theory. For example, one recent study (Arsenault \& Buchsbaum, 2015) grouped the English consonants into five place of articulation "features" (labial, dental, alveolar, palatoalveolar, and velar); in contrast, a typical phonological analysis of English would treat the dental, alveolar, and palatoalveolar consonants as members of a single class of "coronal" consonants, with differences between dental $/ \theta \mathrm{\partial} /$, alveolar $/ \mathrm{s} \mathrm{z/, \text {and }}$ palatoalveolar $/ \int 3 /$ fricatives encoded with additional features such as "strident" (which groups /s z $\int 3 /$ together) or "distributed" (which groups / $\theta$ o $\int 3 /$ together; Hayes, 2009). By ignoring those patterns and treating the dental, alveolar, and palatoalveolar sounds as completely disjoint sets, such studies fail to test the relationship between the neural representation of phonemes and phonological models of structured relationships among phonemes.

An exception to this trend of mismatches between the features used or tested in neuroscience experiments and the features espoused by phonological theory is the work of Lahiri and colleagues (see Lahiri \& Reetz, 2010 for overview). However, their work tests hypotheses about specific phonological contrasts in specific languages, such as the consonant length distinction in Bengali (Roberts, Kotzor, Wetterlin, \& Lahiri, 2014) or vowel features in German (Obleser, Lahiri, $\&$ Eulitz, 2004), but has not thus far tested an entire system of phonological features against recordings of neural activity.

To address these issues, the present study takes a somewhat different approach. First, following evidence that early cortical responses (50-100 ms after stimulus onset) represent encoding of acoustic-phonetic stimulus properties, while later responses (beginning at 100-200 ms after 
stimulus onset) represent more abstract phonological categories (see Salmelin, 2007 for review), parallel analyses are presented of both the entire neural response (incorporating all stages of speech sound processing) and a truncated version of the neural data that excludes the early-stage response. This should eliminate the influence of the early auditory response seen especially with consonant stimuli (e.g., in the temporal response functions in Di Liberto, O’Sullivan, \& Lalor, 2015). Second, rather than testing a specific phonemic contrast, the experiment reported here addresses all consonant phonemes of English (with one exception, /y/, which never occurs word-initially in natural English speech). Finally, this experiment assesses the fit between neural recordings during speech perception and several different feature systems drawn directly from the phonological literature.

\section{MATERIALS AND METHODS}

An overview of the analysis pipeline is given in figure 1. Briefly, syllables were presented auditorily while recording EEG from the listener. EEG data were epoched around each presented syllable, underwent dimensionality reduction, and were labelled with the phonological feature values of each syllable's consonant. Those labels were used to train a set of phonological feature classifiers (one for each phonological feature in the system), and the performance of the system of classifiers was combined to create a confusion matrix summarising the similarity of neural responses to the different consonants, as well as the degree to which the phonological features defined by the given system capture those patterns of similarity.

\section{Stimulus design}

Auditory stimuli were recordings of isolated consonant-vowel (CV) syllables from four American English talkers (2 male, 2 female). Recordings were made in a sound-attenuated booth at a sampling frequency of $44.1 \mathrm{kHz}$ and 16-bit sample precision. During recording, syllables were presented orthographically on a computer screen; example words with target syllables highlighted were also presented to disambiguate orthographic representations as necessary. All consonant phonemes of English were used except $/ \mathrm{y} /$ (which is phonotactically restricted to coda position in English); in all syllables the vowel spoken was /a/. Several repetitions of the syllable list were recorded, to ensure clean recordings of each syllable type.

The recordings were segmented by a phonetician to mark the onset and offset of each CV syllable, as well as the consonant-vowel transition. Recordings were then highpass filtered using a fourth-order Butterworth filter with a cutoff frequency of $50 \mathrm{~Hz}$ (to remove very low frequency background noise present in some of the recordings). The segmented syllables were then excised into separate WAV files, and root-mean-square normalised to equate loudness across talkers and stimulus identities. Final duration of stimuli ranged from $311 \mathrm{~ms}$ (female 2 $/ \mathrm{ta} /)$ to $677 \mathrm{~ms}($ female $1 / \theta \mathrm{a} /)$.

One male and one female talker were arbitrarily specified as "training" talkers, and the other two as "test" talkers. For the training talkers, 3 recordings of each syllable were included in the stimulus set; for the test talkers, only one token of each syllable was included. Each stimulus token was presented 20 times during the experiment. The imbalance between training and test talkers was used to ensure sufficient training data for the classifiers, and to avoid an uncomfortably long experiment duration. Total number of syllables presented was 23 consonants $\times(3$ training tokens +1 test token $) \times 2$ talker genders $\times 20$ repetitions $=3680$ 
A

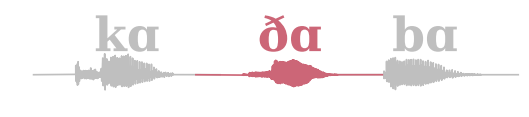

B
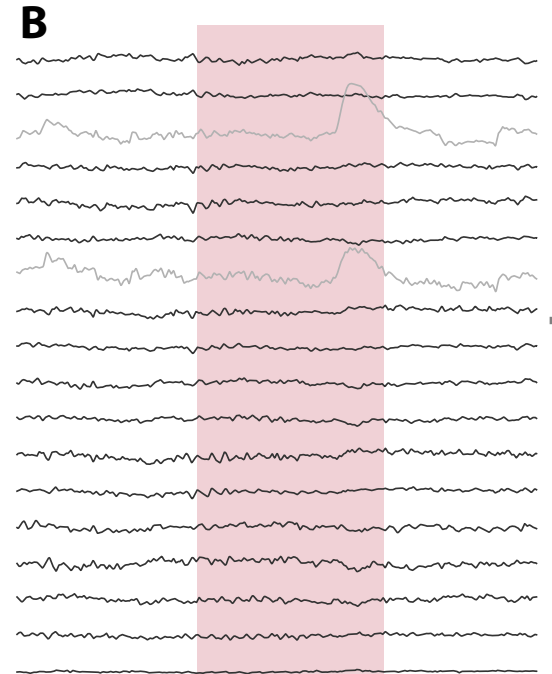

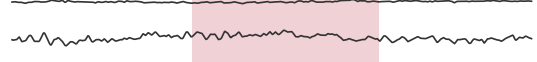
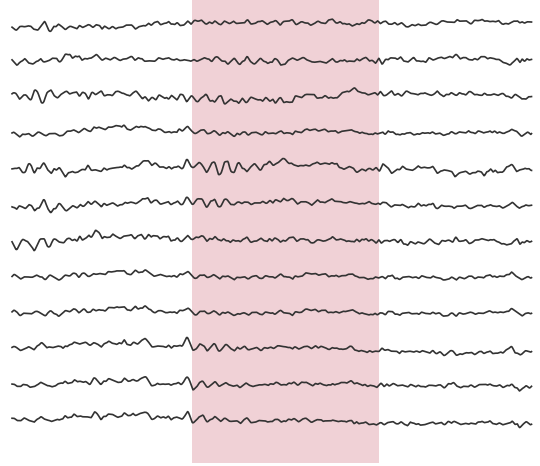

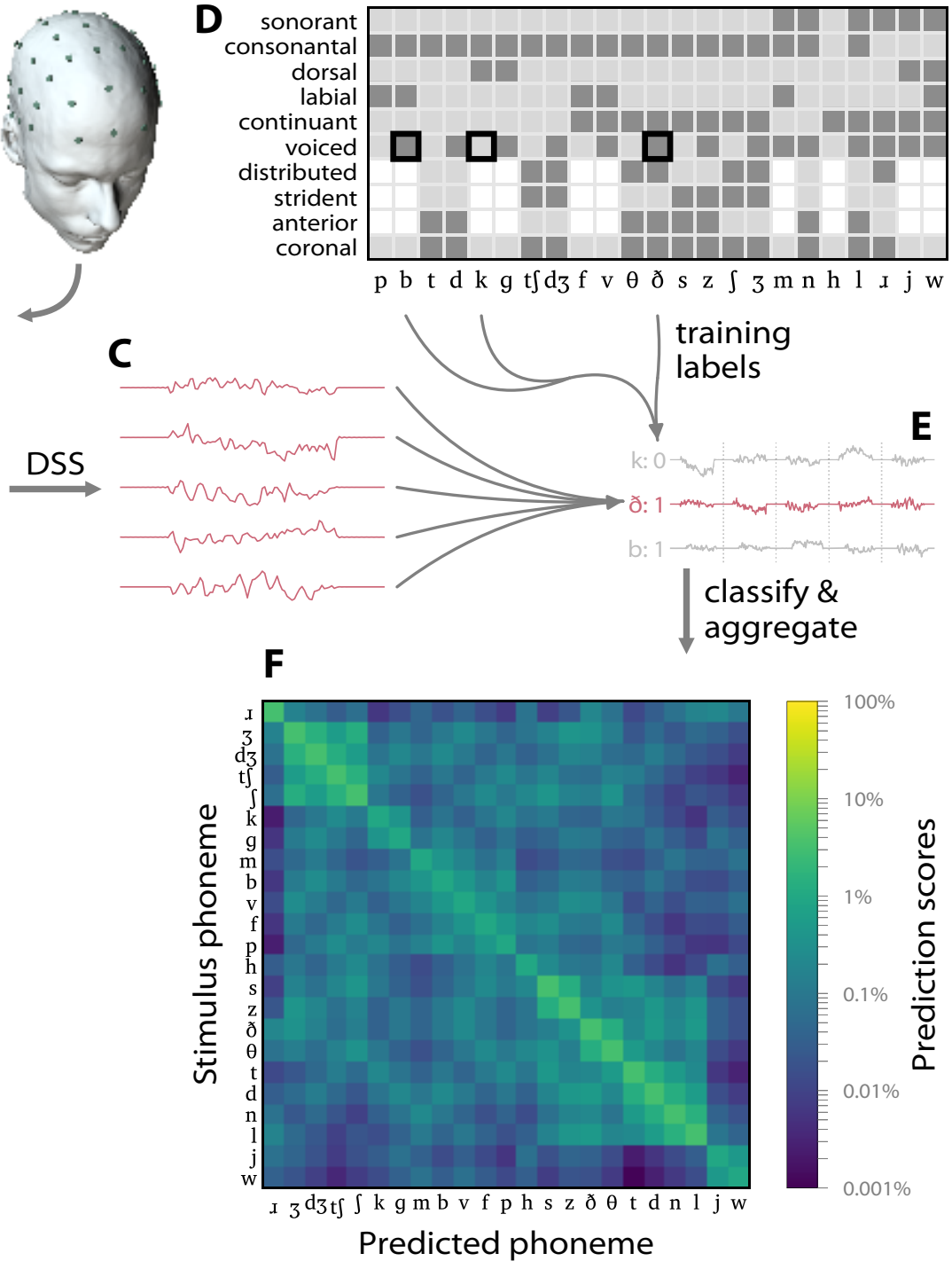

Figure 1: Methods overview. A: portion of a stimulus waveform presented to one subject, with one stimulus highlighted; layout of EEG sensors is shown at right. B: corresponding EEG recording with middle epoch highlighed. Light-colored channels are "bad" channels for this subject, and are excluded from processing (hence they show large deflections due to blinks, which have been regressed out of the "good" channels). C: the highlighted epoch from panel B after Denoising Source Separation (DSS) to reduce dimensionality across channels. D: phonological feature matrix, with heavy boxes indicating values for the three syllables shown in panel A for a given feature ("voiced"). E: DSS signals after concatenation, labelled with the "voiced" feature values from panel D and ready for classification. The process is repeated for each of the phonological features (rows) in panel D. F: confusion matrix made by aggregating classification results across all phonological features in the phonological system. 
stimulus presentations. Additionally, intermixed with the English syllables were a smaller number of similar monosyllabic stimuli spoken by native speakers of four other languages; those data are not analyzed or discussed further here.

\section{Participants}

Twelve listeners (9 female, 19-67 years, median 26) were recruited for this experiment, and were paid an hourly rate for participation. All participants had normal audiometric thresholds $(20 \mathrm{~dB}$ $\mathrm{HL}$ or better at octave frequencies from $250 \mathrm{~Hz}$ to $8 \mathrm{kHz}$ ). All procedures were approved by the University of Washington Institutional Review Board.

\section{Procedure}

Stimuli were delivered through a TDT RP2 real-time processor (Tucker Davis Technologies, Alachula, FL) via Etymotic ER-2 insert earphones, at a level of $65 \mathrm{~dB}$ SPL. Inter-stimulus interval was varied uniformly between 300 and $800 \mathrm{~ms}$ to avoid rhythmicity of the stimulus presentation, which could induce anticipatory listening effects. Stimuli were presented in a unique random order for each listener. The listening task was passive; no verbal, button-press, or other response was required of the participant.

To forestall boredom, listeners were shown cartoons during presentation of the auditory stimuli. The cartoons were episodes of Shaun the Sheep [Starzak \& Sadler (2007); 6-7 minutes in duration] edited to remove the opening and closing credits, and presented without audio or subtitles (the cartoon does not normally include any dialog, so the plots are easy to follow without subtitles or sound). Cartoon episode order was randomised for each participant. Auditory stimulus blocks were timed to correspond to the duration of the cartoons. Listeners were given the chance to take breaks between each block, and encouraged to sit still during each block to reduce motion artifacts in the EEG recording.

\section{Data acquisition}

During stimulus presentation, EEG signals were continuously recorded using a BrainVision 32-channel ActiChamp system at $1000 \mathrm{~Hz}$ sampling frequency. Electrode placement followed a modified form of the standard 10-20 montage, with electrode TP9 moved to A1 (earlobe reference) and electrodes FT9 and FT10 moved to POO9h and POO10h from the 10-05 montage (for compatibility with concurrent studies using shared equipment). Prior to each stimulus, a unique binary identifier of stimulus identity was sent from the presentation computer to the EEG acquisition computer; a second signal was sent from the TDT RP2 to the acquisition computer, synchronised to the stimulus onset. This allowed reliable confirmation of stimulus identity and timing during post-processing.

\section{Data cleaning}

EEG signal processing was carried out in python using the mne-python module (Gramfort et al., 2013). Raw EEG traces were manually inspected and annotated for bad channels, and for spans of time with gross movement artifacts. For a given subject, 0 to 3 channels were marked "bad" and excluded from further processing. Subsequently, a blink detection algorithm was applied to the signal from forehead electrode FP1 (or in one case FP2, because FP1 was extremely noisy for that subject). Annotation to exclude segments of the recording was repeated until the algorithm labels were in close agreement with experimenter-judged blinks. Blinks were then extracted in 1-second epochs centered around the peak, which were used to generate a signal-space 
projection (SSP) operator (Uusitalo \& Ilmoniemi, 1997) for each subject that separated blink-related activity from activity due to other sources. For consistency, the same number of SSP components (four) was used to remove blink artifacts across all subjects. Before applying SSP projectors, the data were mean-subtracted and bandpass-filtered using zero-phase FIR filters (windowed design, Hamming window, 33001 taps), with cutoffs at $0.1 \mathrm{~Hz}$ and $40 \mathrm{~Hz}$ and transition bandwidths of $0.1 \mathrm{~Hz}$ and $10 \mathrm{~Hz}$ (full-width half-maximum of the filter's impulse response $=12 \mathrm{~ms}$ ).

Next, epochs were created around stimulus onsets, and baseline-corrected to yield zero mean for the $100 \mathrm{~ms}$ immediately preceding stimulus onset. Prior annotations of the signal were ignored at this stage, but epochs with absolute voltage changes exceeding $75 \mu \mathrm{V}$ in any channel (excluding channels previously marked as "bad") were dropped. Across subjects, between 3.6\% and $8.8 \%$ of the 3680 English syllable presentations were dropped.

Retained epochs were time-shifted to align on the consonant-vowel transition time instead of the stimulus onset. This was done because information about consonant identity is encoded in the first $\sim 100 \mathrm{~ms}$ of following vowels (Öhman, 1965), so temporal alignment of this portion of the EEG response should hopefully improve the ability of classifiers to learn consonant features. After this re-alignment, epochs had a temporal span from $-258 \mathrm{~ms}$ (the onset of the longest consonant), to $+733 \mathrm{~ms}$ (200 ms after the end of the longest vowel), with $t=0$ fixed at the consonant-vowel transition point. The duration and alignment of each stimulus are visualised in the supplementary material, Figure S2.

At this stage, an optional truncation of the epochs was performed. In one case, the remainder of the analysis was carried out with the entire temporal span of each epoch; in another case, the epochs were truncated to eliminate the brain response prior to $t=100 \mathrm{~ms}$ (in other words, the truncated epochs began $100 \mathrm{~ms}$ after the consonant-vowel transition point of the stimulus). Since the brain's representation of spectrotemporal and acoustic-phonetic features of the stimulus is thought to occur in the first $50-100 \mathrm{~ms}$ after stimulus onset, this truncation should have the effect of eliminating that initial response, and thereby restricting subsequent analysis to the portions of the brain's response that are thought to reflect abstract phonological representations.

\section{Dimensionality reduction}

Retained, transition-aligned epochs were downsampled from $1000 \mathrm{~Hz}$ to $100 \mathrm{~Hz}$ sampling frequency to speed further processing. Spatial dimensionality reduction was performed with denoising source separation (DSS; Särelä \& Valpola, 2005; de Cheveigné \& Simon, 2008). DSS creates orthogonal virtual channels (or "components") that are linear sums of the physical electrode channels, constrained by a bias function (typically the average evoked response across trials of a given type) that orients the components to maximally recover the bias function. In this case a single per-subject bias function was used, based on the average of all epochs for that subject. This is a different strategy than is typically seen in the literature, where separate bias functions are used for each experimental condition. The strategy was chosen to eliminate a possible source of variability in classifier performance: if computing separate bias functions for each consonant or for each consonant token, there would be potentially different numbers of trials being averaged when computing the bias function for the different consonants / tokens. This could lead to different SNRs for the DSS components of different consonants, in a way that 
might bias the classifiers or make certain classifiers' tasks easier than others. An alternative approach would have been to equalise the trial counts for each consonant prior to DSS analysis, but this was rejected due to the exploratory nature of this research (i.e., since the overall amount of data needed was not known, more trials with processing akin to "shrinkage toward the mean" was deemed preferable to fewer trials with a potentially more powerful processing strategy).

Five DSS components were retained, replacing the 28-31 retained electrode channels with 5 orthogonal DSS components for each subject. The number of components retained was the largest number needed so as to include the "knee" point of a scree plot of the relative signal power of the DSS components for each subject's data. Temporal dimensionality reduction was also applied using PCA, to reduce collinearity of (adjacent) temporal samples. Such collinearity can make it difficult for a classifier to learn a decision boundary from the training data. For 6 of the 12 subjects, this reduced the 107 time points to 100 or 101 time points; for the remaining 6 subjects there was no reduction. Finally, the 5 DSS components for each epoch were unrolled into a one-dimensional vector to be passed to the classifiers.

\section{Supervised learning}

At this point, data for one subject comprises a numeric matrix of $\sim 3400$ rows (one row $=$ one retained epoch or "trial") and $\sim 500$ columns (5 unstacked DSS components of $\sim 100$ time points each). Recall that $3 / 4$ of the total trials are from training talkers, so $\sim 2500$ trials are available to train classifiers, employing various labelling and partitioning strategies; the remaining $\sim 900$ trials are then used to assess the information that can be recovered from the neural signals. Three approaches to classifying the neural data were used; for referential ease these are referred to as analyses 1-3.

In analysis 1, each trial is labelled with the consonant that was presented during that epoch, and a classifier was trained to discriminate between each pair of consonants (for 23 consonants, this yields 253 pairwise comparisions, with each comparison having 220 trials of training data). This initial analysis serves as a "sanity check" that sufficient information about the neural processing of consonant identity is picked up by EEG, and that the information remains in the neural signals after all preprocessing steps have been carried out.

In analysis 2, trials are again labelled using consonant identity, but instead of pairwise classifiers, 23 one-versus-rest (OVR) classifiers were trained, each receiving the full set of training data and learning to identify a different "target" consonant. This serves as a baseline condition against which the phonological-feature-based classifiers can be compared.

In analysis 3, trials were labeled with phonological feature values of the consonant heard on that trial. One classifier was trained for each phonological feature, and three different feature systems were explored: the system described in Jakobson, Fant, and Halle's Preliminaries to Speech Analysis (PSA; Jakobson, Fant, \& Halle, 1952), the system in Chomsky and Halle's Sound Pattern of English (SPE; Chomsky \& Halle, 1968), and the system used in the PHOIBLE database (Moran, McCloy, \& Wright, 2013), which is a corrected and expanded version of the system described in Hayes's Introduction to Phonology (2009). The three feature systems each require a different number of features to encode all English consonant contrasts (PSA uses 9, SPE uses 11, and PHOIBLE uses 10), but all represent a reduction of the 23-way multiclass classification problem into a smaller number of binary classification problems. Feature value assignments in 
the three feature systems are illustrated in figure 2 .

All three analyses were carried out separately on the full temporal span of each trial epoch, and on the truncated version of the data. However, the first two analyses (pairwise and OVR classification) yielded results that were broadly similar for the full and truncated epochs, and the differences between the full and truncated results were uninformative, so for brevity only the results based on the full epochs are presented.

In all three analyses, logistic regression classifiers were used, as implemented in scikit-learn (Pedregosa et al., 2011). Stratified 5-fold cross-validation over the training data was employed at each point in a grid search for hyperparameter $\mathrm{C}$ (regularisation parameter; small $\mathrm{C}$ yields smoother decision boundary). The grid search was quite broad; C ranged logarithmically (base 2) from $2^{-5}$ to $2^{16}$. After grid search, each classifier was re-fit on the full set of training data, using the best hyperparameters. The trained classifiers were then used to make predictions about the class of the consonant on each held-out test data trial; in analyses 1 and 2 those class predictions were consonant labels; in analysis 3 those class predictions were phonological feature values.

Because many phonological feature values are unevenly distributed (e.g., of the 23 consonants in the stimuli, only 6 are "+strident"), a custom score function was written for the classifiers that moved the decision boundary threshold from 0.5 to a value that equalised the ratios of incorrect classifications (i.e., equalised false positive and false negative rates), minimised this value (called the "equal error rate"), and returned a score of 1 - equal error rate. This precludes the situation where high accuracy scores are achieved by simply guessing the more numerous class in most or all cases. Since such class imbalance can occur in any of the analyses (and is guaranteed to happen for the one-versus-rest classifiers in analysis 2), a score function that minimised equal error rate was used for all analyses. Additionally, many phonological feature systems involve some sparsity (i.e., some features are undefined for certain phonemes; for example, in the PSA feature system, the feature "nasal" is unvalued for phonemes $/ \mathrm{h} 1 \mathrm{j} \mathrm{j} \mathrm{w} /$ ). In such cases, trials for phonemes that are undefined on a given phonological feature were excluded from the training set for that classifier.

\section{Aggregation of classifier results}

At this point in analysis 3, data for one subject comprises a matrix of $\sim 900$ rows (1 per test trial) and 9-11 columns (1 per phonological feature classifier; number of columns depends on which phonological feature system is being analyzed). Each cell of that matrix is a 0 or 1 classification for that combination of trial and phonological feature. From these data, the accuracy of each classifier (subject to the same equal-error-rate constraint used during training) was computed. Next, a $23 \times 23 \times \mathrm{N}$ array was constructed (where 23 is the number of consonants in the stimuli, and $\mathrm{N}$ is the number of phonological features in the current system, i.e., between 9 and 11). The first dimension represents which consonant was presented in the stimulus, and the second dimension represents the possible percepts. For each plane along the third axis (i.e., for a given phonological feature classifier), the cells of the plane are populated with either the accuracy or the error rate ( 1 minus the accuracy) of that classifier, depending on whether the consonant indices of the row and column of that cell match (accuracy) or mismatch (error rate) in that feature. For example, a cell in row /p/, column /b/ of the voicing classifier plane would have as its entry the error rate of the voicing classifier, signifying the probability that a voiceless $/ \mathrm{p} /$ would be mis-classified as a voiced /b/. 
PSA (9 features)

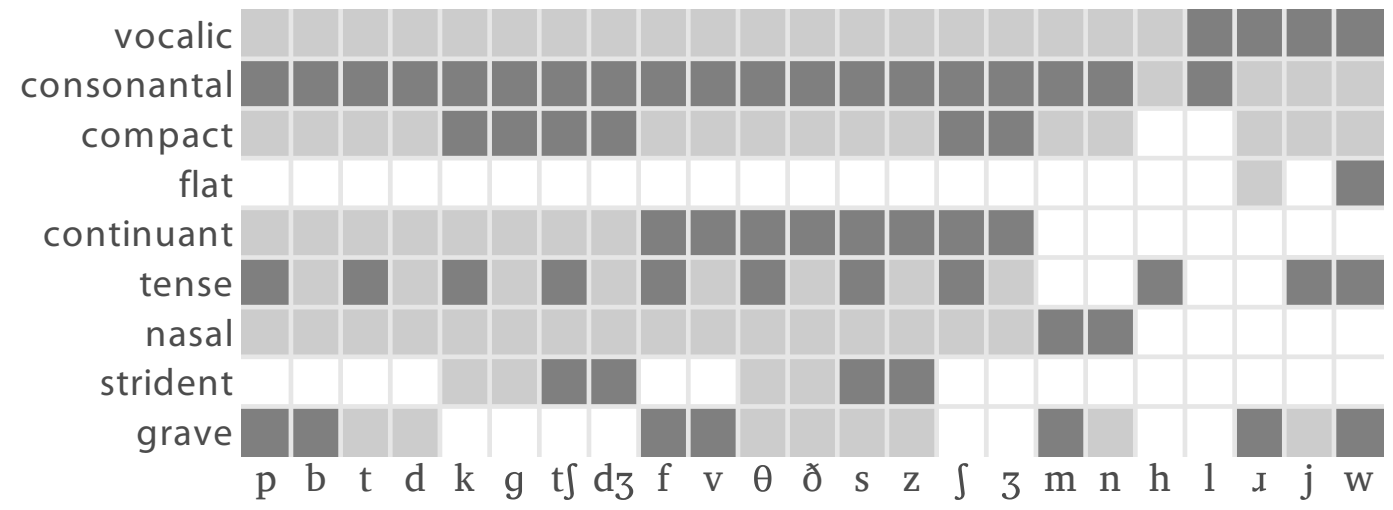

SPE (11 features)
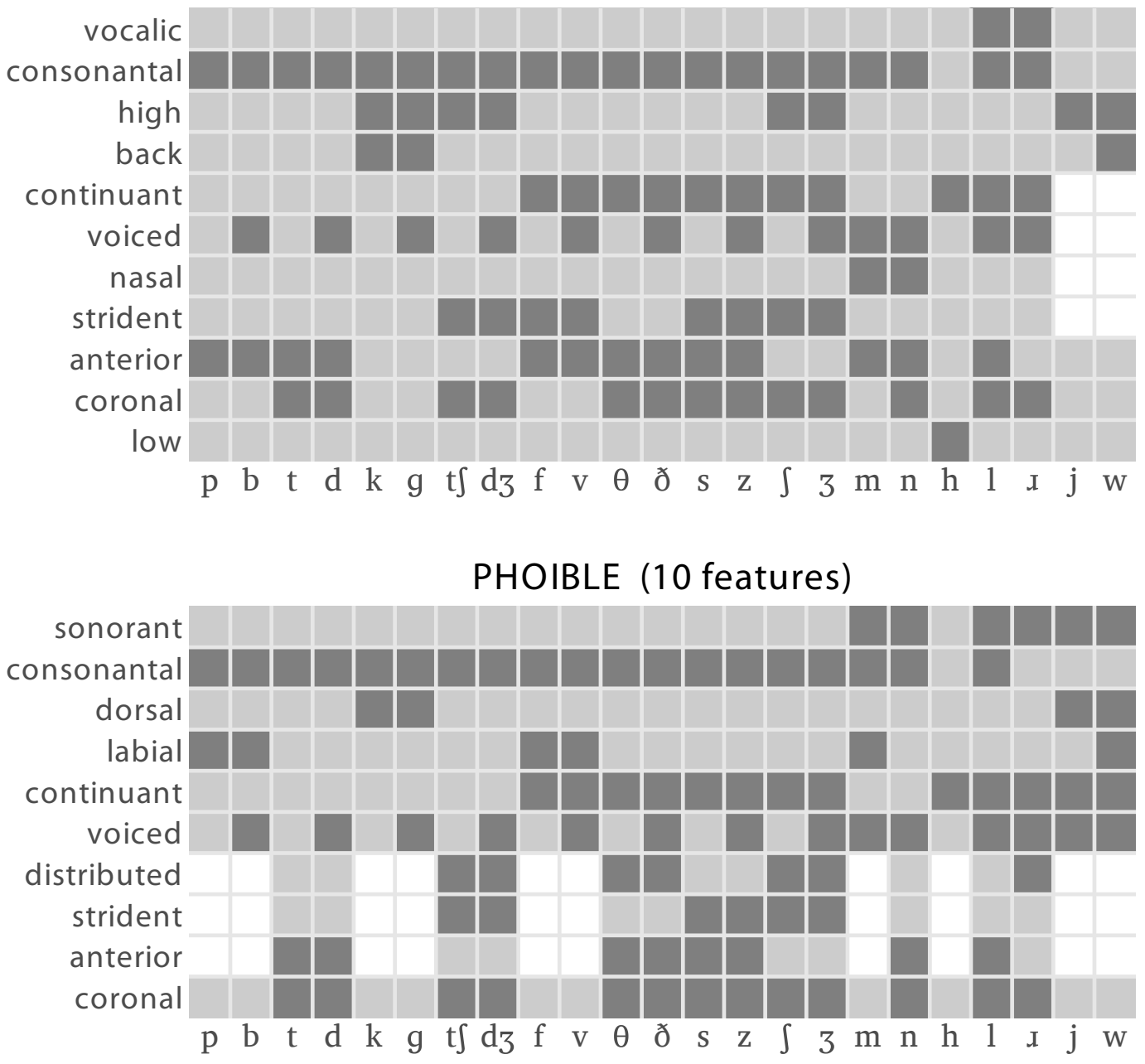

Figure 2: Feature matrices for the three feature systems used in analysis 3. Dark gray cells indicate positive feature values, light gray cells indicate negative feature values, and white cells indicate phoneme-feature combinations that are undefined in that feature system. The names of the features reflect the original sources; consequently the same feature name may have different value assignments in different systems. 
Finally, the feature planes are collapsed by taking the product along the last dimension, yielding the joint probability that the input consonant (the stimulus) would be classified as any given output consonant (the percept). For features involving sparsity, cells corresponding to undefined feature values were given a chance value of 0.5 (results were broadly similar when such cells were simply excluded from the computation). The resulting $23 \times 23$ matrices can be thought of as confusion matrices: each cell gives the probability that the bank of phonological feature classifiers in that feature system would classify a heard consonant (given by the row label) as a particular consonant percept (given by the column label).

In such matrices, a perfectly performing system would be an identity matrix: values of 1 along the main diagonal, and values of 0 everywhere else. To quantify the extent to which each confusion matrix resembles an identity matrix, a measure of relative diagonality was computed as in Equation 1, where $\mathrm{A}$ is the matrix, $i$ is an integer vector of row or column indices (starting at 1 in the upper-left corner), and $w$ is a vector of ones of the same length as $i$.

(1)

$$
\text { diagonality }=\frac{w \mathrm{~A} w^{\mathrm{T}} \times i \mathrm{~A} i^{\mathrm{T}}-i \mathrm{~A} w^{\mathrm{T}} \times w \mathrm{~A} i^{\mathrm{T}}}{\sqrt{w \mathrm{~A} w^{\mathrm{T}} \times i^{2} \mathrm{~A} w^{\mathrm{T}}-\left(i \mathrm{~A} w^{\mathrm{T}}\right)^{2}} \times \sqrt{w \mathrm{~A} w^{\mathrm{T}} \times w \mathrm{~A}\left(i^{2}\right)^{\mathrm{T}}-\left(w \mathrm{~A} i^{\mathrm{T}}\right)^{2}}}
$$

Briefly, this is an adaptation of the Pearson correlation for samples. It computes the correlation between the matrix's rows and columns, which reflects the fraction of the total mass of the matrix that lies on the main diagonal. This measure yields a value of 1 for an identity matrix, zero for a uniform matrix, and -1 for a matrix with all its mass on the minor diagonal. A derivation of this measure is provided in the supplementary material.

This notion of diagonality requires that adjacent rows be relatively similar and distant rows be relatively dissimilar (and likewise for columns); otherwise, the formula's notion of distance between rows (or columns) cannot be justifiably applied. Therefore, before computing diagonality, the matrices were submitted to a hierarchical clustering of the rows using the optimal leaf ordering algorithm (Bar-Joseph, Gifford, \& Jaakkola, 2001) as implemented in scipy (Jones, Oliphant, Peterson, \& others, 2017), and the column order was permuted to match the optimal ordering of the rows.

\section{RESULTS}

\section{Analysis 1: Pairwise classifiers}

Results (aggregated across subjects) for the pairwise classifiers are shown in figure 3. Because the classifier score function imposed an equal-error-rate constraint, there is no difference between, e.g., proportion of $/ \mathrm{p} /$ trials mistaken for $/ \mathrm{b} /$ and proportion of $/ \mathrm{b} /$ trials mistaken for $/ \mathrm{p} /$, so the upper triangle is omitted.

In general, the mean accuracy across subjects for a given pairwise comparison was always above 90\% (differences along the diagonal in Figure 3 are numerically small and hence hard to distinguish in color); individual accuracy scores for each subject were generally above $80 \%$ and are shown in the supplementary material, figure S5. These plots indicate that consonant identity can be recovered fairly well from brain responses to the stimuli. However, a suite of pairwise classifiers is not a particularly realistic model of how speech perception is likely to work: during normal comprehension it isn't generally the case that listeners are always choosing between 1 of 

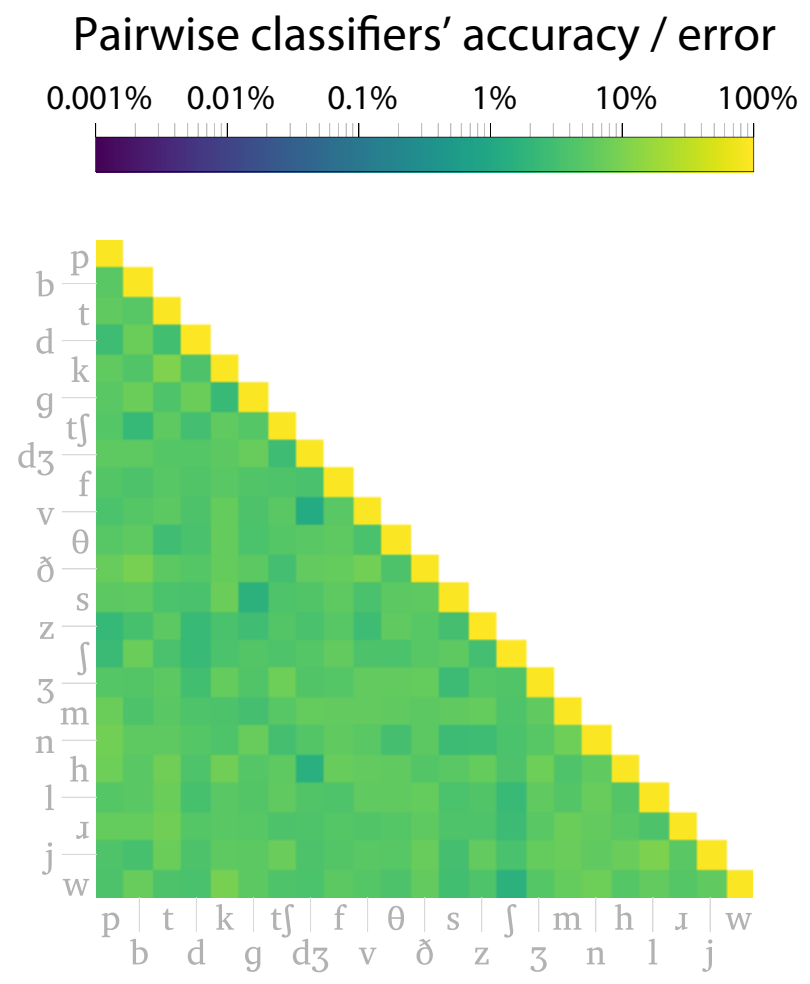

Figure 3: Across-subject average accuracy/error for pairwise classifiers. Off-diagonal cells represent the error rates for the pairwise classifier indicated by that cell's row/column labels; diagonal cells represent the mean accuracy for all pairs in which that consonant is one element. 


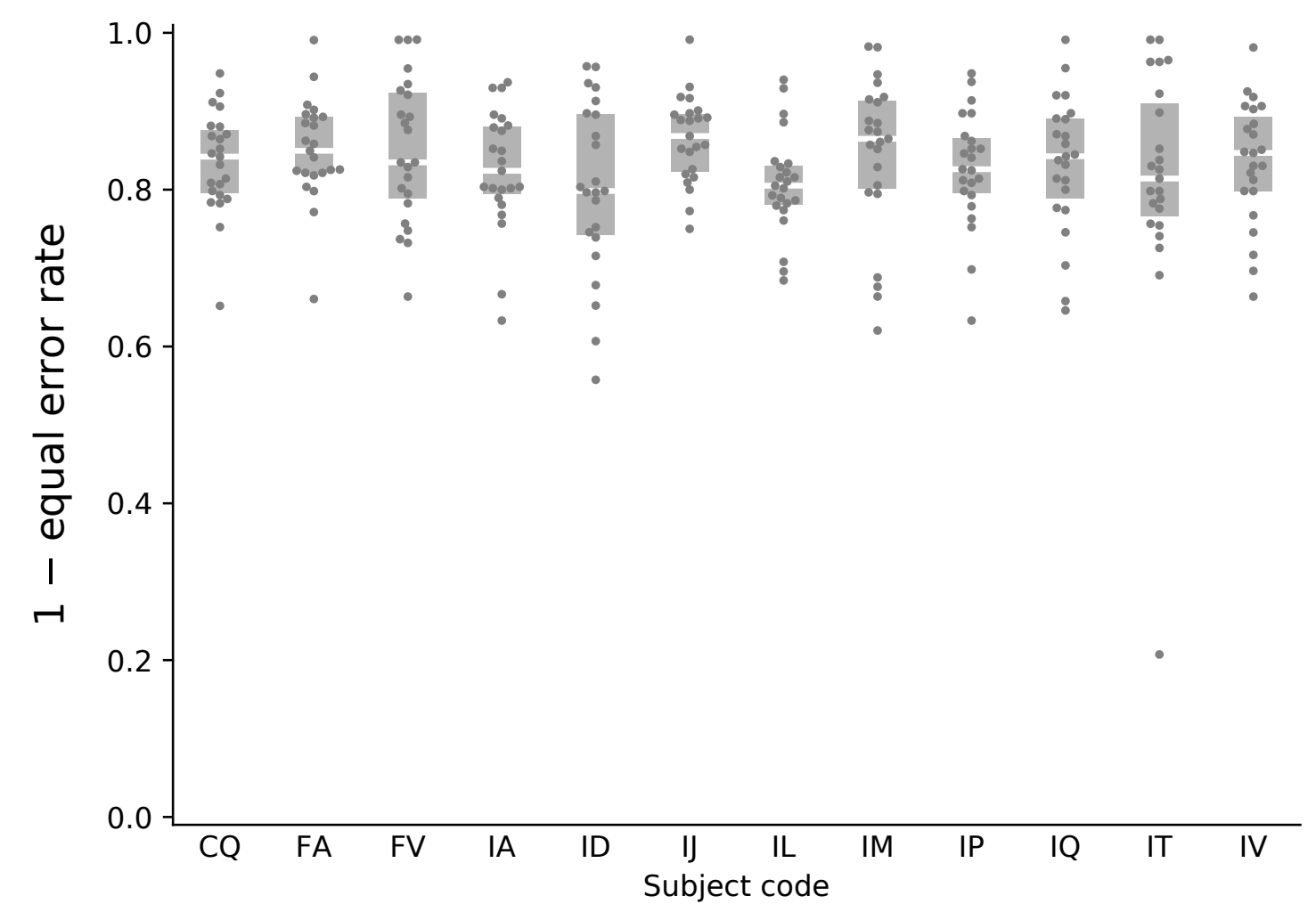

Figure 4: Within-subject distributions of accuracy for one-versus-rest classifiers. Boxes show quartiles; dots are individual classifier accuracies.

2 options for "what that consonant was." Rather, consonant identification is a closed-set identification task: listeners know the set of possible consonants they might hear, and must determine which one was actually spoken. Analysis 2 provides a more realistic model of this scenario.

\section{Analysis 2: OVR classifiers}

Results for the OVR classifiers in analysis 2 are shown in figures 4 and 5 . As a reminder, these classifiers learn to discriminate brain responses to one particular consonant against all other consonants as a group (which is a harder problem than pairwise comparison, as the non-target class is much more heterogeneous). Unsurprisingly, the OVR classifiers were not as good as the pairwise classifiers at identifying consonants from the EEG data; accuracies ranged from below chance to near-perfect on individual subject data, with first quartiles between $74 \%$ and $82 \%$ and third quartiles between $83 \%$ and $92 \%$ (see figure 4 ).

Moreover, despite passable accuracy scores, the classifiers don't actually learn much, classifying both non-target and target trials as the target consonant at similar rates (i.e., little within-column differentiation in figure 5A). The faint checkerboard pattern in the upper left quadrant of the matrix does indicate that, e.g., the /b/ classifier in the second column is 

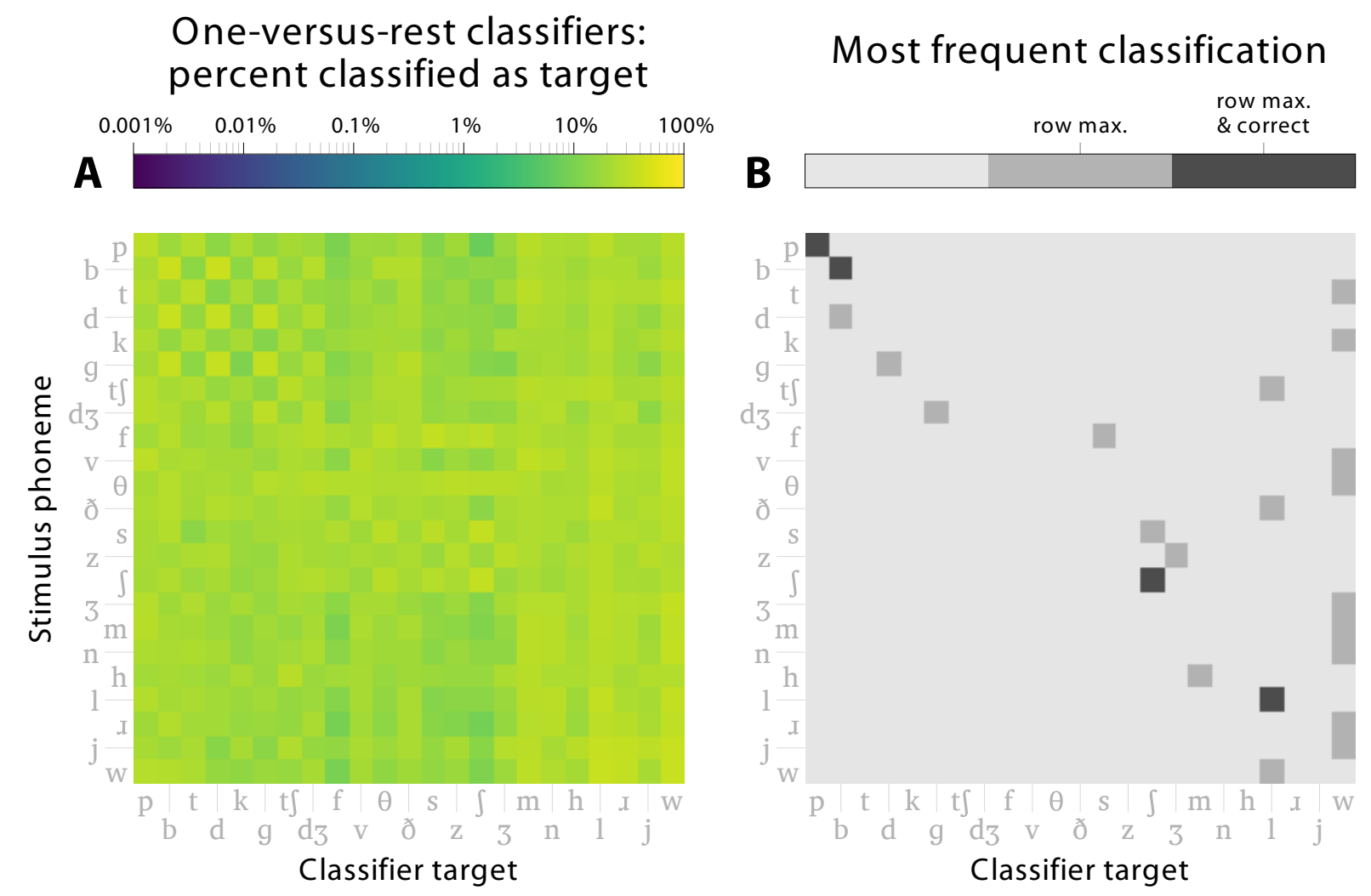

Figure 5: Results for one-versus-rest classifiers, aggregated across subjects. Each column represents a single classifier, with its target class indicated by the column label. Row labels correspond to the test data input to each classifier. A: cells on the diagonal represent the ratio of true positive classifications to total targets (also called "hit rate" or "recall"); off-diagonal elements represent the ratio of false positive classifications to total non-targets ("false alarm rate") for the consonant given by the row label. B: most frequent classification of each stimulus consonant, emulating across-classifier voting. Consonants that are correctly identified are indicated by dark gray cells along the main diagonal; consonants that are most frequently incorrectly identified are medium-gray cells.

relatively more likely to mistakenly classify trials with $/ \mathrm{d} /$ or $/ \mathrm{g} /$ stimuli as $/ \mathrm{b} /$ trials, than it is to make the same mistake on trials with $/ \mathrm{p} /, / \mathrm{t} /$, or $/ \mathrm{k} / \mathrm{stimuli}$. There is also some indication that classifiers for fricative consonants (especially /f s z $\int 3 /$ ) tended to make more misclassifications to trials with (non-target) fricative stimuli, compared to trials with non-fricative stimuli (the broad vertical stripe near the middle of the matrix, which is darker in its upper and lower thirds). However, the classifiers' abilities to make these basic discriminations of voiced versus voiceless stop consonants or fricatives versus non-fricatives is still rather poor (e.g., the /b/ classifier marks $19 \%$ of /p/ trials as /b/, and only $41 \%$ of /b/ trials as /b/). Finally, looking across classifiers for a given stimulus phoneme, it is rarely the case that the most frequent classification is the correct one (cf. lack of diagonal elements in figure 5B).

\section{Analysis 3: Phonological feature classifiers}

Whereas analyses 1 and 2 test classification of neural signals based on identity of the consonant in each stimulus, analysis 3 tests classification of the same signals based on phonological feature 


\section{PSA feature-based classifiers' prediction scores}

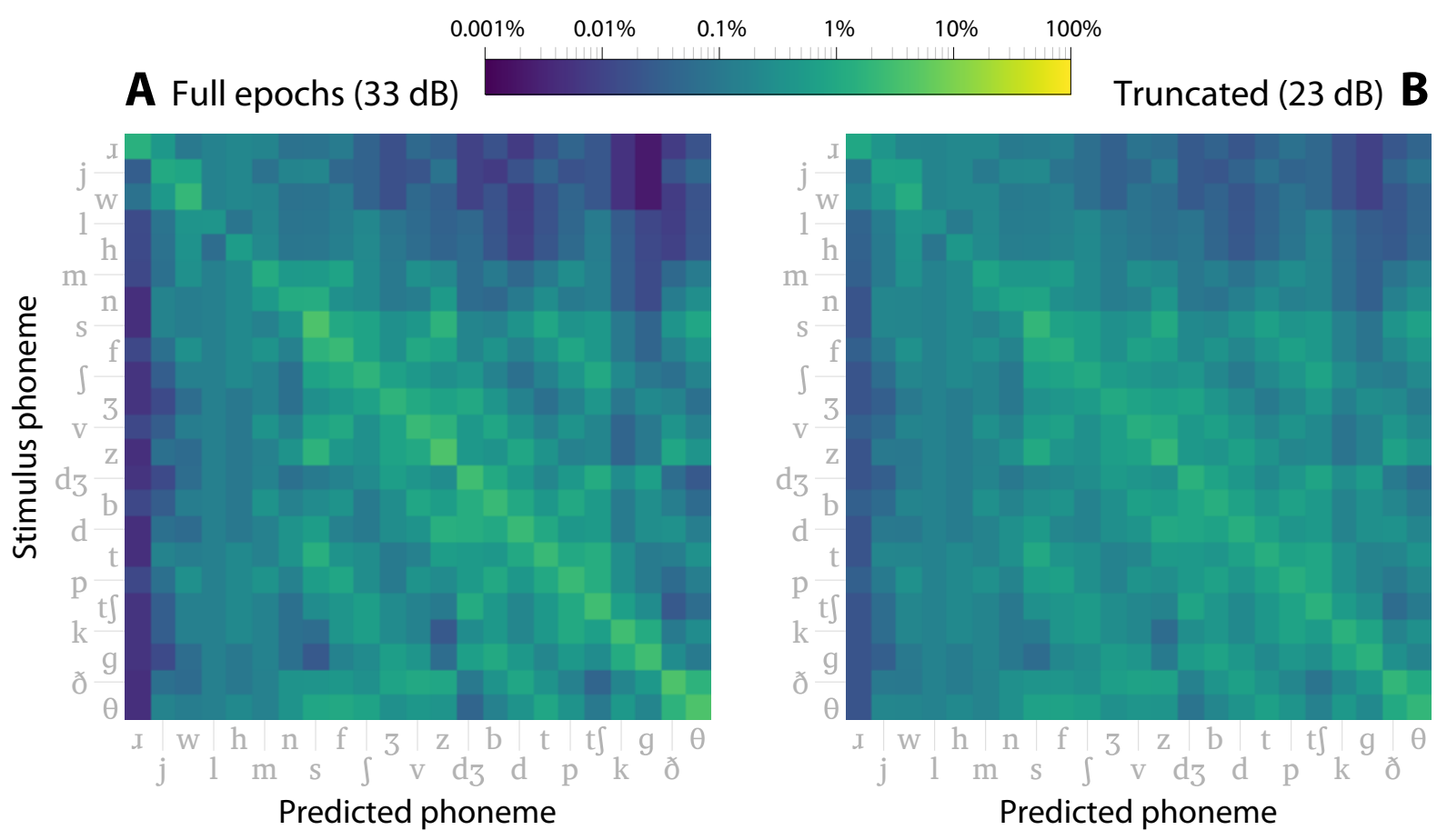

Figure 6: Confusion matrices derived from the PSA phonological feature classifiers, based on A: the full temporal span of each epoch, or B: truncated epochs. Row labels correspond to the test data input to each classifier. Notable features include fairly reliable identification of $/ \mathrm{x} /$ trials, and relatively uniform confusability of the "+consonantal, -nasal" consonants (everything below and to the right of $/ \mathrm{s} /$ ). The dynamic ranges are given in parentheses above each matrix; there is roughly an order-of-magnitude decrease when using the truncated epochs.

values of those consonants, and classifications of test data are aggregated across systems of phonological features to yield consonant-level confusion matrices similar to those seen in figures 3 and 5 . The results of this aggregation for the three phonological feature systems tested (PSA, SPE, and PHOIBLE) are shown in figures 6, 7, and 8, respectively, with each figure showing confusion matrices based on both full and truncated epochs (plots for individual subject's data can be seen in the supplementary material, Figures S6-S8). Unlike the prior confusion matrices, where rows and columns followed a standard order based on consonant manner of articulation, in figures 6,7, and 8 the matrices are ordered based on a hierarchical clustering of the rows (performed separately for each feature system) using optimal leaf ordering. Therefore, the row and column orders do not necessarily match across the three figures (though it is consistent between panels within each figure), so attention to the row and column labels is necessary when visually comparing the matrices across feature systems. A version of the full-epochs confusion matrix for each feature system with consistent row/column order across feature systems is given in the supplementary material, Figure S9.

Compared to the confusion matrices for pairwise (figure 3) and OVR (figure 5A) classifiers, the magnitude of the values in the phonological-feature-based confusion matrices is smaller (the 
highest classification scores tend to be around 2-4\%; this arises because each cell is a product of several probability values, which naturally makes the resulting numbers smaller). However, the smallest values are a few orders of magnitude smaller than the values in the pairwise and OVR confusion matrices, making the distinction between similar and dissimilar cells more apparent. Consequently, much more structure is apparent in the phonological-feature-based confusion matrices. For example, figure 6A shows that when information is combined from the 9 feature classifiers in the PSA system, $2 \times 2$ submatrices for voiced-voiceless consonant pairs $/ \partial \theta /$ and $/ \mathrm{g}$ $\mathrm{k}$ / are visible in the lower-right corner, suggesting that the classifier trained to discriminate voicing (encoded by the "tense" feature in the PSA system) was relatively less accurate than other phonological feature classifiers in that system (perhaps because PSA's "tense" feature groups voiceless consonants together with $/ \mathrm{j} /$ and /w/, which may be an unnatural grouping as far as the brain is concerned).

As another example, consider the first and third columns of Figure 6A, showing the rate at which all stimuli are classified as either $/ \mathrm{I} /$ or $/ \mathrm{w} /$, respectively. Consonants other than $/ \mathrm{x} /$ are very unlikely to be classified as $/ \mathrm{x} /$, whereas consonants other than $/ \mathrm{w} /$ are much more likely to get mis-classified as /w/. This result is probably a consequence of how $/ \mathrm{x} /$ and $/ \mathrm{w} /$ are encoded in the PSA system: a single feature "flat" distinguishes $/ \mathrm{I} /$ from $/ \mathrm{w} /$ and is unvalued for all other consonants, so the "flat" classifier is trained only on brain responses to $/ \mathrm{x} /$ and $/ \mathrm{w} /$, and when presented with test stimuli from all other consonants turns out to be much more likely to classify them as +flat (/w/-like) than as -flat (/I/-like).

Interestingly, the general pattern of confusions is preserved when classifiers learn from the truncated epochs (Figure 6B) rather than the full temporal span of the neural response to each syllable (Figure 6A), though the overall dynamic range of the confusion matrix is reduced by roughly an order of magnitude. Nonetheless, combining information from all the phonological feature classifiers in the PSA system still usually results in the correct classification being the dominant one (the highest classification score in each row is the diagonal element), for all consonants except /n/ when using the full epochs, and for all consonants except /n/ and /l/ when using the truncated epochs.

Looking across feature systems, similar $2 \times 2$ submatrices of poorly discriminated consonant pairs are seen in figure 7 (e.g., /x l/ and /m n/) and figure 8 (e.g., /s z/ and /j w/), and both SPE and PHOIBLE show a $4 \times 4$ submatrix in the upper left quadrant corresponding to the post-alveolar consonants $/ \int 3 \mathrm{t} \int \mathrm{d} 3 /$, suggesting that, in addition to the voicing distinction, the fricative-affricate distinction (encoded by the "continuant" feature in both systems) was not well learned by the classifiers. Additionally, the PHOIBLE system shows a large block in the lower right quadrant corresponding to /t d $\theta \partial \mathrm{s} \mathrm{z} 1 \mathrm{n} /$ (the "+anterior" consonants in that system), again suggesting that the partitioning of phonemes encoded by that feature is less easily recoverable from the neural signals than other features in the PHOIBLE system.

The confusion matrices for the SPE and PHOIBLE systems also show the same general confusion pattern in the full-epoch and truncated-epoch analyses, and show similar reductions in dynamic range as the PSA system did. Finally, it is interesting to note that the pair $/ \mathrm{j} w /$ is poorly discriminated by the classifiers in all three systems, although the distinction is encoded by different features in each: "grave" in PSA, "back" in SPE, and "labial" in PHOIBLE. 


\section{SPE feature-based classifiers' prediction scores}

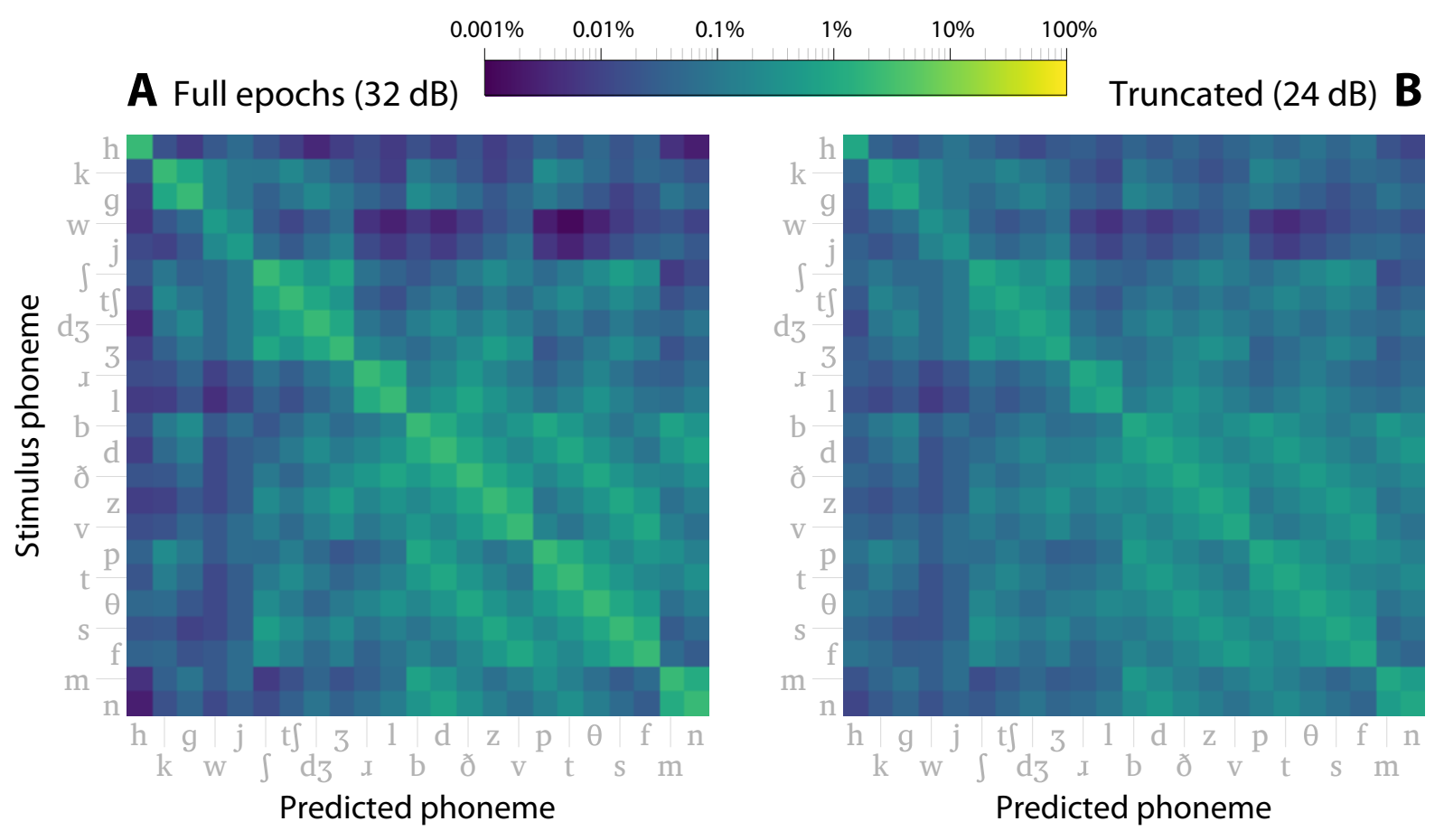

Figure 7: Confusion matrices derived from the SPE phonological feature classifiers, based on A: the full temporal span of each epoch, or B: truncated epochs. Notable features include the $4 \times 4$ block of post-alveolar fricatives and affricates in the upper left quadrant, the reliable identification of $/ \mathrm{h} /$, and several $2 \times 2$ submatrices indicating confusible pairs of consonants (e.g., $/ \mathrm{g} \mathrm{k} /, / \mathrm{d} \mathrm{l} /$, and $/ \mathrm{m} \mathrm{n} /$ ). The dynamic ranges are given in parentheses above each matrix; there is roughly an order-of-magnitude decrease when using the truncated epochs. 


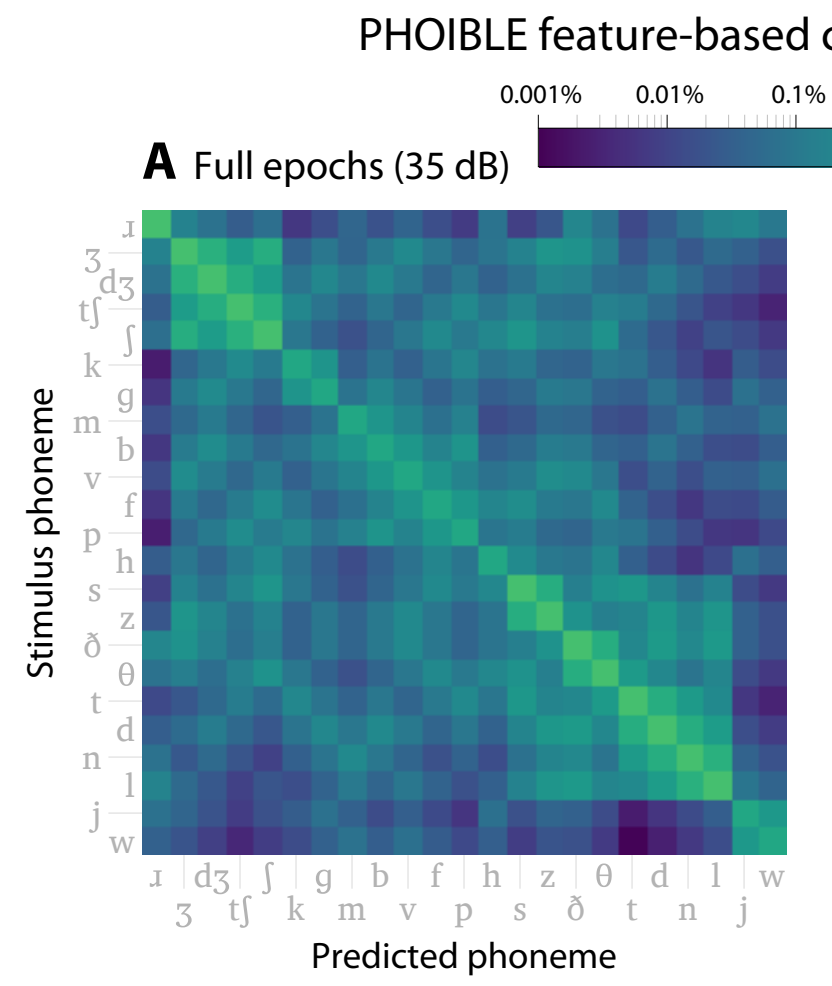

\section{PHOIBLE feature-based classifiers' prediction scores}

\begin{tabular}{llll}
$0.1 \%$ & $1 \%$ & $10 \% \quad 100 \%$ \\
\hline
\end{tabular} Truncated (24 dB) B

Figure 8: Confusion matrices derived from the PHOIBLE phonological feature classifiers, based on A: the full temporal span of each epoch, or B: truncated epochs. Notable features include the $4 \times 4$ block of post-alveolar fricatives and affricates in the upper left, the $8 \times 8$ block of anterior alveoloar consonants in the lower right (with $2 \times 2$ voiced-voiceless submatrices $/ \mathrm{s} \mathrm{z/}$ and $/ \partial \theta /$ within it), and the relative distinctiveness of $/ \mathrm{j} \mathrm{w} /$ from all other consonants, but not from each other. The dynamic ranges are given in parentheses above each matrix; there is roughly an order-of-magnitude decrease when using the truncated epochs. 


\section{Full epochs Truncated epochs}

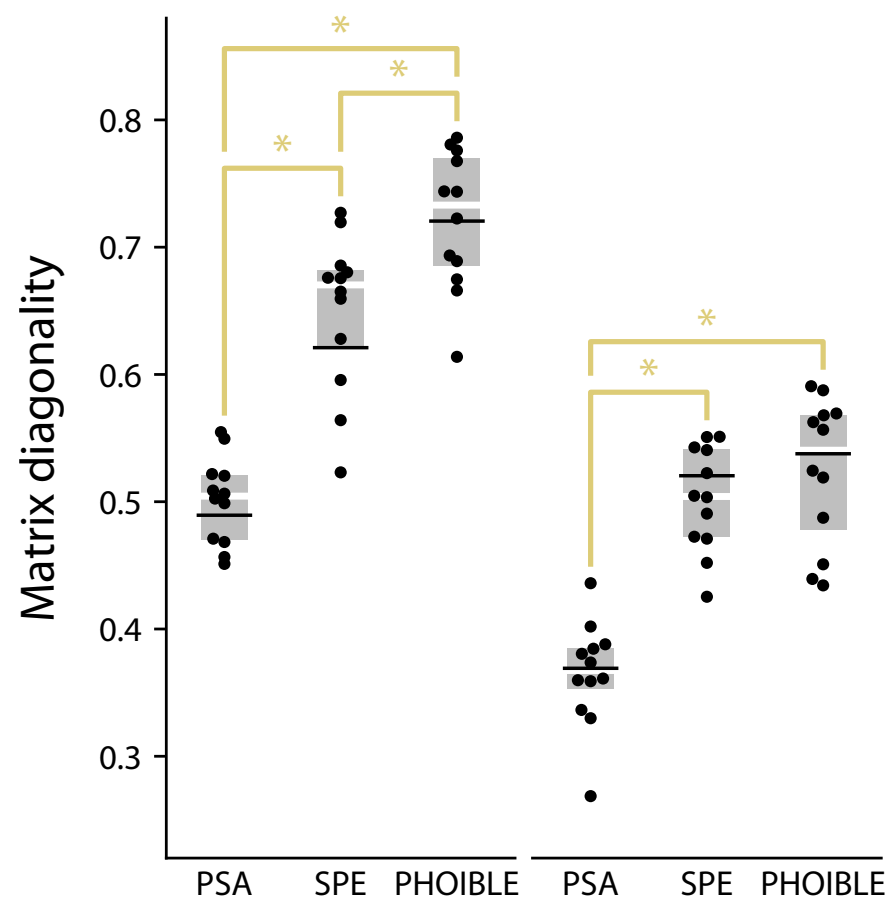

Figure 9: Matrix diagonality measures for each of the three feature systems tested, for analyses based on the full temporal span of each epoch (left panel) or the truncated epochs (right panel). Gray boxes show quartiles; circles represent diagonality measures for individual subject data, and black horizontal lines represent the diagonality measures for the across-subject average matrices shown in figures 6, 7, and 8 . Brackets indicate significant differences between feature systems (paired-samples t-tests, bonferroni-corrected, all corrected p-values $<0.01$ ).

To quantify the degree to which the neural responses reflect the contrasts encoded by each feature system, we compute the diagonality of each matrix (the degree to which the mass of the matrix falls near the main diagonal). Matrix diagonality values for each subject's data, along with the across-subject average matrices, are shown in Figure 9. The PHOIBLE feature system fares considerably better than the PSA and SPE feature systems on this measure, suggesting that the contrasts encoded by the PHOIBLE system as a whole more closely reflect the kinds of information extracted by the brain during speech processing and subsequently detected in the EEG signals. Unsurprisingly, the diagonality is substantially reduced for all three systems when computed based on the truncated epochs, but the general trend is more or less preserved: PSA is still far and away the worst fit to the neural data, but the difference between SPE and PHOIBLE is less pronounced and fails to reach statistical significance in the analysis of truncated data. To ensure that the diagonality measure was not merely reflecting differences in signal quality for different subjects (i.e., due to scalp conductivity, EEG cap fit, etc.), we regressed subjects' diagonality scores in each feature system against the signal-to-noise ratio of their epoched EEG data, and found no evidence to support a correlation (see supplementary material, figures S3 and S4).

To determine which features contributed most to this measure of diagonality, we performed a 


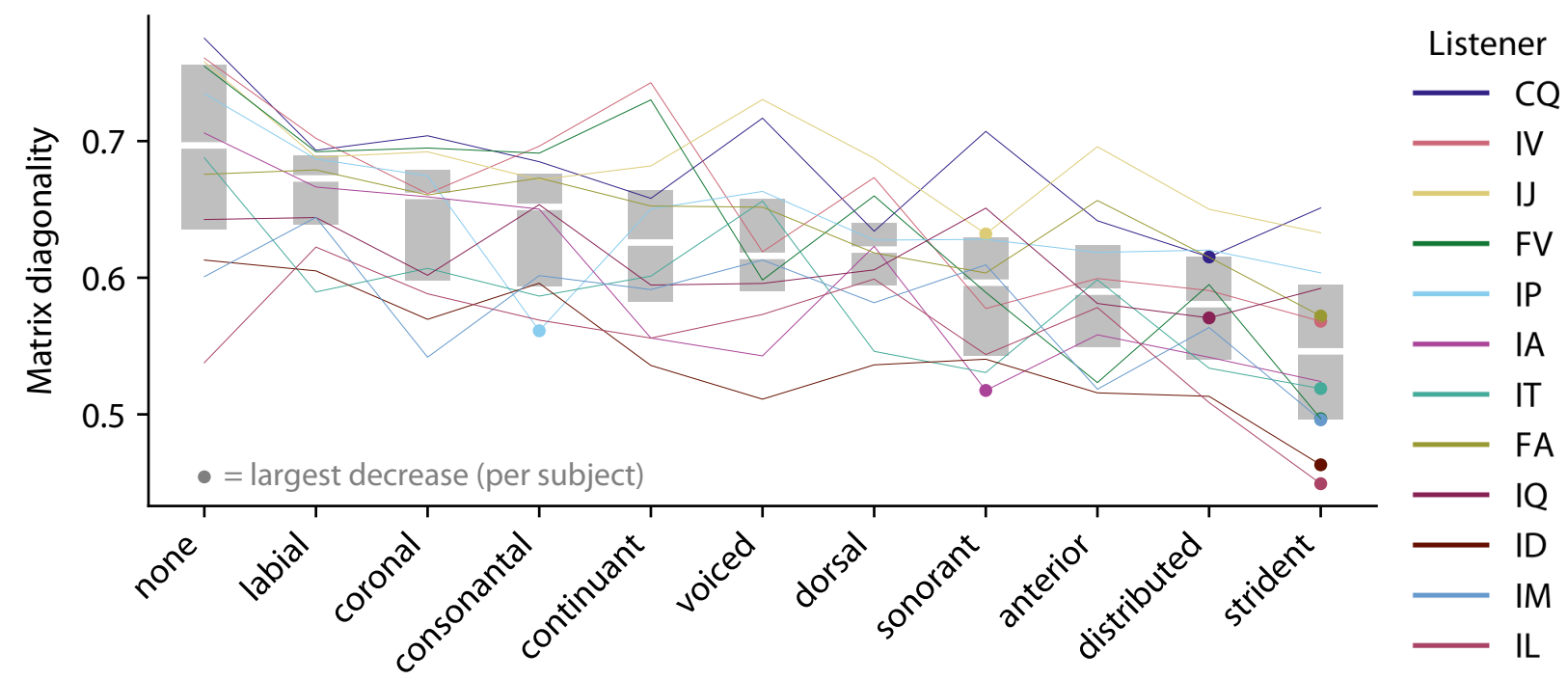

Figure 10: Leave-one-out analysis of feature influence on the diagonality measure, for the PHOIBLE feature classifiers, based on the full temporal span of each epoch. Abscissa labels indicate which feature was excluded; connected points reflect diagonality values for a given listener; boxes show quartiles across listeners for each left-out feature. For each listener, the lowest diagonality is indicated by a large dot; 7 of the 12 listeners showed the greatest decline in diagonality when omitting the "strident" feature.

leave-one-out analysis, in which each feature was excluded in turn from the computation of the confusion matrix, hierarchical clustering with optimal leaf ordering was performed, and the resulting diagonality was calculated. This analysis was done on the individual listeners' confusion matrices, rather than on the average confusion matrix across listeners, in order to assess cross-subject agreement in the rank ordering of feature importance. Results of this analysis for the PHOIBLE feature system are shown in figure 10. There appears to be considerable heterogeneity in the rank ordering of feature importance across subjects, evidenced by the lack of monotonicity in the connecting lines for several of the subjects in figure 10 . Nonetheless, most subjects (7 of 12) showed the greatest decrease in diagonality when omitting the "strident" feature, which distinguishes /s z $\int 3 \mathrm{t} \int \mathrm{d}_{3} /$ from the rest of the coronal consonants

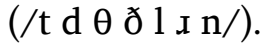

\section{DISCUSSION}

This paper describes a technique for comparing theoretical accounts of phonological features to recordings of brain activity during speech perception, and illustrates the technique with three phonological feature systems drawn from the linguistic literature. The approach uses machine learning classifiers to model the representation of abstract classes of speech sounds by the brain, and combines information across classifiers to construct predicted patterns of similarity or confusion among phonemes. This work differs from many past studies of phonological feature representation in its emphasis on phonological feature systems drawn from the linguistic literature, and in its attempt to model the entire consonant inventory of a language rather than a few select contrasts.

Of course, languages comprise vowels as well as consonants, and a natural extension of this 
work would model the vowels as well. Moreover, the phonemic contrasts present in English are only a subset of the attested contrasts in the world's languages, and another natural extension would be to apply these techniques to modeling the brain activity of native listeners of a variety of languages (to explore the representation of lexical tone, voice quality contrasts, ejective and implosive consonants, etc., and to characterise any differences in representation that emerge across different languages). Finally, a thorough test of abstract phonological representation should at least account for patterns of allophony (e.g., the differing pronunciations of $/ \mathrm{t} / \mathrm{in}$ different positions in a word). Whereas these analyses did include multiple tokens of each consonant from multiple talkers, they did not model allophonic variation, and doing so is another natural extension of this work (either with polysyllabic stimuli or continuous speech).

One way in which this study does address the question of abstract representations is the parallel analysis of both the full temporal span and a truncated span of the brain response to each syllable. Specifically, the fact that the patterns of confusion for each phonological feature system are basically preserved when analyzing the truncated epochs suggests that the early brain response around 50-100 ms post-stimulus onset (associated with acoustic-phonetic stimulus properties) is not strictly necessary to recover (some of) the patterns of similarity predicted by each phonological feature system. Therefore, to the extent that confusion matrices differ across feature systems in the analysis of truncated data, we can more confidently infer differences in the fidelity of each feature system's representation of phonological information, as opposed to patterns of phonetic similarity. However, it is important to remember that late-stage processing of consonantal information is concurrent with early-stage processing of incoming vowel information as the stimulus unfolds through time. Therefore, the influence of acoustic cues to consonant identity that are carried by the vowel cannot be entirely ruled out even in our truncated data. By truncating at $100 \mathrm{~ms}$ after the consonant-vowel transition, we have hopefully minimised or eliminated the influence of formant transitions during the vowel onset, but other cues (such as vowel duration covarying with the voicing status of preceding stop consonants) cannot be fully ruled out by truncation alone.

Perhaps the most promising aspect of this work is its potential to provide complementary information to behavioral studies of phoneme confusion (e.g., the classic study of Miller \& Nicely, 1955). In such studies, confusions are induced by degrading the stimuli (usually by adding background noise), which has unequal effects on different speech sounds due to variations in their cue robustness (Wright, 2001, 2004). In contrast, this technique estimates phoneme confusion based on brain responses to unmodified natural speech, not speech-in-noise, thereby removing biases related to cue robustness. Of course, the nature of the auditory system, the brain, and EEG also favors some speech sound contrasts over others. For example, EEG relatively faithfully reflects the total energy of acoustic stimuli, making it ideal for tracking things like the amplitude envelope of an utterance (Power, Foxe, Forde, Reilly, \& Lalor, 2012), but this also ought to favor phoneme-level contrasts based on differences in rise time (e.g., fricative-affricate distinctions like $/ \mathrm{S} /$ versus $/ \mathrm{t} \int /$ ) compared to purely spectral contrasts such as $/ \mathrm{f} /$ versus $/ \theta /$. Therefore, failure of a classifier to recover a given contrast in the neural recordings should not be taken as definitive evidence that the brain does not represent that particular abstraction - such failures may arise from the limitations of our measurement tools.

Nevertheless, even if we can't rule out any particular feature being represented in the brain 
based on these data alone, we can still say that the abstractions implied by a system with a better fit to the neural data are likely a closer approximation of a listener's phonological knowledge than the abstractions implied by a system with poor fit to the neural data. Even such coarse insights can provide a foundation for follow-up studies that focus on specific features or contrasts, and drilling down on the patterns of errors made by each classifier may reveal specific shortcomings of particular feature systems that could guide their refinement (e.g., whether the "consonantal" feature includes $/ \mathrm{I} /$, as in SPE, or does not, as in PSA and PHOIBLE; or whether sparsity in the assignment of feature values improves or degrades the fit between phonological feature system and neural data).

Finally, because we used only acoustically natural speech stimuli, the patterns of neural representation seen in this work may not be fully divorced from patterns of similarity in the acoustic properties of the stimuli (as mentioned above). As discussed in the introduction, Evans and Davis (2015) used a mixture of acoustically natural and synthetic stimuli that partially addresses this worry (though their approach also fails to remove duration-related cues), and found no evidence of phonological feature representation after accounting for stimulus acoustics in that way (though with a fairly limited stimulus set). Here, our data seem to indicate patterns of speech processing that are at least consistent with the brain extracting information analogous to phonological features, but further work is needed to definitively establish such a claim. Nevertheless, even if the information learned by the classifiers in this study is partially acoustic in nature, knowing which phonological feature system best recapitulates those patterns still helps to build a bridge between the neuroscience of language and traditional phonological theory.

\section{Future directions}

As mentioned above, natural extensions of this work are expanding the stimulus set to include vowels, other languages, and continuous speech, and testing listeners of other language backgrounds. Several other researchers have made inroads in these directions already, especially with regard to continuous speech (e.g., Lalor \& Foxe, 2010; Di Liberto \& Lalor, 2017; Di Liberto et al., 2015; Khalighinejad, da Silva, \& Mesgarani, 2017; Mesgarani et al., 2014). In addition, there are other theories of phonological representation that could be assessed using this technique, such as articulatory phonology (Browman \& Goldstein, 1989, 1992), or the featurally underspecified lexicon model (Lahiri \& Reetz, 2002, 2010). There is also the potential to derive a system of features directly from the neural data, without reference to linguistic theory, and assess its similarities to published accounts post hoc.

From a methodological perspective, different preprocessing or classification strategies might improve the clarity of the confusion matrices by reducing classification errors that are strictly due to noise, as opposed to those that reflect a genuine mistake about which sounds ought to pattern together. Other imaging modalities (such as MEG or combined EEG/MEG) could also increase signal and thereby improve our ability to resolve differences between models.

Finally, our earliest application of this technique was actually as a model of how speech sounds from an unfamiliar language are filtered through native phonological categories (Hasegawa-Johnson et al., 2017), as part of a larger project involving non-traditional approaches to automatic speech recognition. Much work remains to realise the potential of that application as well. 


\section{ACKNOWLEDGMENTS}

Special thanks to Mark Hasegawa-Johnson and Ed Lalor who were instrumental in the early development of this technique, and to Nick Foti, Bryan Gick, Alex Gramfort, Kathleen Hall, Eric Larson, Majid Mirbagheri, and Doug Pulleyblank for helpful and stimulating discussions about various aspects of the project along the way.

\section{DISCLOSURE STATEMENT}

The authors report no conflict of interest.

\section{FUNDING}

This work was supported in part by the NIDCD under grant T32DC005361.

\section{REFERENCES}

Arsenault, J. S., \& Buchsbaum, B. R. (2015). Distributed neural representations of phonological features during speech perception. Journal of Neuroscience, 35(2), 634-642. https://doi.org/10.1523/JNEUROSCI.2454-14.2015

Bar-Joseph, Z., Gifford, D. K., \& Jaakkola, T. S. (2001). Fast optimal leaf ordering for hierarchical clustering. Bioinformatics, 17(Suppl 1), S22-S29. https://doi.org/10.1093/bioinformatics/17.suppl_1.S22

Bromberger, S., \& Halle, M. (1989). Why phonology is different. Linguistic Inquiry, 20(1), 51-70. Retrieved from http://www.jstor.org/stable/4178613

Browman, C. P., \& Goldstein, L. (1989). Articulatory gestures as phonological units. Phonology, 6(02), 201-251. https://doi.org/10.1017/S0952675700001019

Browman, C. P., \& Goldstein, L. (1992). Articulatory phonology: An overview. Phonetica, 49(3-4), 155-180. https://doi.org/10.1159/000261913

Chomsky, N., \& Halle, M. (1968). The sound pattern of English. Cambridge, MA: MIT Press.

de Cheveigné, A., \& Simon, J. Z. (2008). Denoising based on spatial filtering. Fournal of Neuroscience Methods, 171(2), 331-339. https://doi.org/10.1016/j.jneumeth.2008.03.015

Di Liberto, G. M., \& Lalor, E. C. (2017). Indexing cortical entrainment to natural speech at the phonemic level: Methodological considerations for applied research. Hearing Research, 348, 70-77. https://doi.org/10.1016/j.heares.2017.02.015

Di Liberto, G. M., O’Sullivan, J. A., \& Lalor, E. C. (2015). Low-frequency cortical entrainment to speech reflects phoneme-level processing. Current Biology, 25(19), 2457-2465. https://doi.org/10.1016/j.cub.2015.08.030

Evans, S., \& Davis, M. H. (2015). Hierarchical organization of auditory and motor representations in speech perception: Evidence from searchlight similarity analysis. Cerebral Cortex, 25(12), 4772-4788. https://doi.org/10.1093/cercor/bhv136 
Gramfort, A., Luessi, M., Larson, E. D., Engemann, D. A., Strohmeier, D., Brodbeck, C., ... Hämäläinen, M. S. (2013). MEG and EEG data analysis with MNE-Python. Frontiers in Neuroscience, 7(267), 1-13. https://doi.org/10.3389/fnins.2013.00267

Hasegawa-Johnson, M., Jyothi, P., McCloy, D. R., Mirbagheri, M., di Liberto, G., Das, A., ... Lee, A. K. C. (2017). ASR for under-resourced languages from probabilistic transcription. IEEE/ACM Transactions on Audio, Speech, and Language Processing, 25(1), 50-63. https://doi.org/10.1109/TASLP.2016.2621659

Hayes, B. P. (2009). Introductory phonology. Oxford: Wiley-Blackwell.

Hickok, G., Okada, K., \& Serences, J. T. (2009). Area Spt in the human planum temporale supports sensory-motor integration for speech processing. Fournal of Neurophysiology, 101(5), 2725-2732. https://doi.org/10.1152/jn.91099.2008

Jakobson, R., Fant, C. G. M., \& Halle, M. (1952). Preliminaries to speech analysis: The distinctive features and their correlates. Cambridge: MIT Press.

Jones, D. (1957). The history and meaning of the term "phoneme”. In W. E. Jones \& J. Laver (Eds.), Phonetics in Linguistics: A Book of Readings (pp. 187-204). London: Longman.

Jones, E., Oliphant, T., Peterson, P., \& others. (2017). SciPy: Open source scientific tools for Python (Version 1.0.0). Retrieved from http://www.scipy.org/

Khalighinejad, B., da Silva, G. C., \& Mesgarani, N. (2017). Dynamic encoding of acoustic features in neural responses to continuous speech. The fournal of Neuroscience, 37(8), 2176-2185. https://doi.org/10.1523/JNEUROSCI.2383-16.2017

Lahiri, A., \& Reetz, H. (2002). Underspecified recognition. In C. Gussenhoven \& N. Warner (Eds.), Laboratory phonology 7 (pp. 637-676). Berlin: Mouton de Gruyter.

Lahiri, A., \& Reetz, H. (2010). Distinctive features: Phonological underspecification in representation and processing. Journal of Phonetics, 38(1), 44-59.

https://doi.org/10.1016/j.wocn.2010.01.002

Lalor, E. C., \& Foxe, J. J. (2010). Neural responses to uninterrupted natural speech can be extracted with precise temporal resolution. European fournal of Neuroscience, 31(1), 189-193. https://doi.org/10.1111/j.1460-9568.2009.07055.x

Leonard, M. K., Bouchard, K. E., Tang, C., \& Chang, E. F. (2015). Dynamic encoding of speech sequence probability in human temporal cortex. Journal of Neuroscience, 35(18), 7203-7214. https://doi.org/10.1523/JNEUROSCI.4100-14.2015

Markiewicz, C. J., \& Bohland, J. W. (2016). Mapping the cortical representation of speech sounds in a syllable repetition task. NeuroImage, 141, 174-190.

https://doi.org/10.1016/j.neuroimage.2016.07.023

Mesgarani, N., Cheung, C., Johnson, K., \& Chang, E. F. (2014). Phonetic feature encoding in human superior temporal gyrus. Science, 343(6174), 1006-1010.

https://doi.org/10.1126/science.1245994 
Mielke, J., \& Hume, E. V. (2006). Distinctive features. Encyclopedia of Language \& Linguistics, 723-731. Retrieved from http://linkinghub.elsevier.com/retrieve/pii/B0080448542000365

Miller, G. A., \& Nicely, P. E. (1955). An analysis of perceptual confusions among some English consonants. The Journal of the Acoustical Society of America, 27(2), 338-352. https://doi.org/10.1121/1.1907526

Moran, S., McCloy, D. R., \& Wright, R. A. (Eds.). (2013). PHOIBLE: Phonetics information base and lexicon online. Munich: Max Planck Digital Library. Retrieved from http://phoible.org

Obleser, J., Lahiri, A., \& Eulitz, C. (2004). Magnetic brain response mirrors extraction of phonological features from spoken vowels. Journal of Cognitive Neuroscience, 16(1), 31-39. https://doi.org/10.1162/089892904322755539

Öhman, S. E. G. (1965). Durations of formant transitions. Speech, Music and Hearing Quarterly Progress and Status Report, 6(1), 10-13. Retrieved from http://www.speech.kth.se/prod/publications/files/qpsr/1965/1965_6_1_010-013.pdf

Pedregosa, F., Varoquaux, G., Gramfort, A., Michel, V., Thirion, B., Grisel, O., ... Duchesnay, E. (2011). Scikit-learn: Machine learning in Python. Fournal of Machine Learning Research, 12, 2825-2830. Retrieved from http://www.jmlr.org/papers/v12/pedregosa11a.html

Power, A. J., Foxe, J. J., Forde, E.-J., Reilly, R. B., \& Lalor, E. C. (2012). At what time is the cocktail party? A late locus of selective attention to natural speech. European Fournal of Neuroscience, 35(9), 1497-1503. https://doi.org/10.1111/j.1460-9568.2012.08060.x

Roberts, A. C., Kotzor, S., Wetterlin, A., \& Lahiri, A. (2014). Asymmetric processing of durational differences - electrophysiological investigations in Bengali. Neuropsychologia, 58, 88-98. https://doi.org/10.1016/j.neuropsychologia.2014.03.015

Salmelin, R. (2007). Clinical neurophysiology of language: The MEG approach. Clin. Neurophysiol., 118(2), 237-254. https://doi.org/10.1016/j.clinph.2006.07.316

Särelä, J., \& Valpola, H. (2005). Denoising source separation. Journal of Machine Learning Research, 6, 233-272. Retrieved from http://www.jmlr.org/papers/v6/sarela05a.html

Starzak, R., \& Sadler, C. (2007). Shaun the Sheep (season 1). Bristol: Aardman Animations.

Uusitalo, M. A., \& Ilmoniemi, R. J. (1997). Signal-space projection method for separating MEG or EEG into components. Medical \& Biological Engineering \& Computing, 35(2), 135-140. https://doi.org/10.1007/BF02534144

Vaden, K. I., Muftuler, L. T., \& Hickok, G. (2010). Phonological repetition-suppression in bilateral superior temporal sulci. NeuroImage, 49(1), 1018-1023.

https://doi.org/10.1016/j.neuroimage.2009.07.063

Wright, R. A. (2001). Perceptual cues in contrast maintenance. In E. V. Hume \& K. Johnson (Eds.), The role of speech perception in phonology (pp. 251-277). San Diego: Academic Press.

Wright, R. A. (2004). A review of perceptual cues and cue robustness. In B. Hayes, R. Kirchner, \& D. Steriade (Eds.), Phonetically based phonology (pp. 34-57). Cambridge: Cambridge University Press. 


\title{
Supplement to "Investigating the fit between phonological feature systems and brain responses to speech using EEG"
}

\author{
Daniel R. McCloy and Adrian K. C. Lee \\ University of Washington, Institute for Learning and Brain Sciences, 1715 NE Columbia \\ Rd., Seattle, WA 98195-7988
}

\section{CONSONANT-VOWEL SEGMENTATION}

In this study, for the purpose of segmentation and alignment the consonant-vowel transition was defined as the onset of prominent formants in syllables with obstruent consonants (stops, fricatives, and affricates), the onset of higher formants (F4, F5) and corresponding waveform amplitude changes in syllables with nasal consonants, and the beginning of formant steady-state (visually and auditorily judged) for syllables with approximant consonants (liquids and glides). Segmentation based on waveform or spectrogram features was supplemented by auditory judgment of the pre- and post-boundary segments as needed.

\section{MATRIX DIAGONALITY}

Here we describe a method of computing the "diagonality" of a matrix. The measure is based on the Pearson correlation for points in the $(x, y)$ plane, and when applied to a non-negative square matrix, yields values between -1 and 1 . To give an intuition of what the results of this diagonality measure will look like: diagonal matrices yield a value of 1 , matrices that are nonzero only on the minor diagonal yield a value of -1 , and uniform matrices yield a value of 0 . The line of reasoning follows and expands on a discussion posted on Mathematics Stack Exchange (Tad, 2015) for computing the correlation between the rows and columns of a square matrix.

Consider a non-negative square matrix A:

$$
\mathrm{A}=\left[\begin{array}{cccc}
0 & 0 & 0 & 0.4 \\
0 & 0 & 0.2 & 0 \\
0 & 0 & 0 & 0 \\
0.3 & 0 & 0 & 0
\end{array}\right]
$$

We can conceive of $\mathrm{A}$ as representing a set of points $(c, r)$, where the coordinates of those points are determined by the column and row indices of the matrix (so for a $4 \times 4$ matrix like $A$, the $(c, r)$ locations are given by $c, r \in\{1,2,3,4\}$ ). For each of those points $(c, r)$, the corresponding entry in A describes the mass of the point at that location. Since 13 of the 16 entries in A are zero, we can represent A in the $c, r$ plane as a set of just 3 points of differing masses. This is shown in figure $\mathrm{S} 1$, with grayscale values and annotations denoting the mass at each location:

Ordinarily, Pearson correlations are computed on a set of points that are equally weighted, so to compute the Pearson correlation for the points in figure S1 we must either incorporate weights into the Pearson formula, or transform our data such that each point is equally weighted. The latter approach can be achieved straighforwardly by scaling our matrix A such that all its entries are integers, and treating those integer entries as the number of replicated, equally-weighted points at 


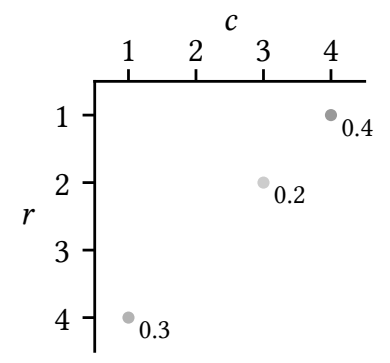

Figure S1: Representation of matrix A in the $c, r$ plane. Note that $r$ increases downwards, to accord with conventional matrix row numbering.

each location. This scaling factor $s$ will be the least common denominator of the fractional representations of the elements of $\mathrm{A}$, which in this case is $10 .{ }^{1}$ Thus we can compute $\mathrm{B}=s \mathrm{~A}$ :

$$
\mathrm{B}=s \times \mathrm{A}=\left[\begin{array}{llll}
0 & 0 & 0 & 4 \\
0 & 0 & 2 & 0 \\
0 & 0 & 0 & 0 \\
3 & 0 & 0 & 0
\end{array}\right]
$$

From B then we can generate a set of (equally weighted) points $\mathrm{P}$, where the coordinates of each point are (column, row) indices, and the number of replicates of each coordinate pair is determined by $\mathrm{B}_{r, c}$ : $\mathrm{P}=[(1,4),(1,4),(1,4),(3,2),(3,2),(4,1),(4,1),(4,1),(4,1)]$

A plot of the points $\mathrm{P}$ will superficially resemble figure S1, because the replicated points will be overplotted and impossible to distinguish. Nonetheless, we can now straightforwardly apply the formula for the Pearson correlation of a sample, given here with coordinates $(c, r)$ replacing the more traditional $(x, y)$ :

$$
\begin{aligned}
\hat{\rho} & =\frac{\operatorname{cov}(c, r)}{\operatorname{stdev}(c) \times \operatorname{stdev}(r)} \\
& =\frac{\sum_{i=1}^{n}\left(c_{i}-\bar{c}\right)\left(r_{i}-\bar{r}\right)}{\sqrt{\sum_{i=1}^{n}\left(c_{i}-\bar{c}\right)^{2}} \times \sqrt{\sum_{i=1}^{n}\left(r_{i}-\bar{r}\right)^{2}}} \\
& =\frac{n \sum_{i=1}^{n} c_{i} r_{i}-\sum_{i=1}^{n} c_{i} \sum_{i=1}^{n} r_{i}}{\sqrt{n \sum_{i=1}^{n} c_{i}^{2}-\left(\sum_{i=1}^{n} c_{i}\right)^{2}} \sqrt{n \sum_{i=1}^{n} r_{i}^{2}-\left(\sum_{i=1}^{n} r_{i}\right)^{2}}}
\end{aligned}
$$

The derivation of equation (3) from equation (2) is provided in a following section, for those interested in the details. We can now translate equation (3) into a form that more directly reflects our matrix B: the term $n$ is the number of points in $\mathrm{P}$, which is also equal to the sum of the entries of $\mathrm{B}$, and the coordinates $c_{i}$ and $r_{i}$ are the column and row indices of each (nonzero) entry of the matrix. For an $m \times m$ matrix, if we define a vector $i=[1 \ldots m]$ representing row or column indices, and a vector $w$ of all ones of the same length as $i$, then we can rewrite the summation terms in equation (3) as follows:

\footnotetext{
${ }^{1}$ Here the entries of A have been chosen to make the scale factor small (so that the set of points $\mathrm{P}$ is of manageable size for illustrative purposes) but in general there is no need for the entries of A to be "tidy" numbers, because, as will become clear below, the scaling factor $s$ will cancel out of the Pearson equation, and we can operate directly on the matrix A without affecting the resulting correlation value.
} 


$$
\begin{array}{rlrl}
n & =w \mathrm{~B} w^{\mathrm{T}} & \sum r_{i}=i \mathrm{~B} w^{\mathrm{T}} & \sum r_{i}^{2}=i^{2} \mathrm{~B} w^{\mathrm{T}} \\
\sum r_{i} c_{i}=i \mathrm{~B} i^{\mathrm{T}} & \sum c_{i}=w \mathrm{~B} i^{\mathrm{T}} & \sum c_{i}^{2}=w \mathrm{~B}\left(i^{2}\right)^{\mathrm{T}}
\end{array}
$$

This yields the following equivalent expression for $\hat{\rho}$ :

$$
\hat{\rho}=\frac{w \mathrm{~B} w^{\mathrm{T}} \times i \mathrm{~B} i^{\mathrm{T}}-i \mathrm{~B} w^{\mathrm{T}} \times w \mathrm{~B} i^{\mathrm{T}}}{\sqrt{w \mathrm{~B} w^{\mathrm{T}} \times i^{2} \mathrm{~B} w^{\mathrm{T}}-\left(i \mathrm{~B} w^{\mathrm{T}}\right)^{2}} \times \sqrt{w \mathrm{~B} w^{\mathrm{T}} \times w \mathrm{~B}\left(i^{2}\right)^{\mathrm{T}}-\left(w \mathrm{~B} i^{\mathrm{T}}\right)^{2}}}
$$

Note here that if we substitute $s \mathrm{~A}$ for B in equation (4), the scaling factor $s$ can be factored out of both numerator and denominator and cancelled, showing that it is not in fact necessary to scale the matrix to integer values in order to compute the correlation between rows and columns using the Pearson formula. Rather, the original values of A act as (fractional) weights on the vectors of row and column indices, in exactly the same fashion as the integer numbers of replicated, superimposed points in the scaled matrix B did:

$$
\begin{aligned}
\hat{\rho} & =\frac{w(s \mathrm{~A}) w^{\mathrm{T}} \times i(s \mathrm{~A}) i^{\mathrm{T}}-i(s \mathrm{~A}) w^{\mathrm{T}} \times w(s \mathrm{~A}) i^{\mathrm{T}}}{\sqrt{w(s \mathrm{~A}) w^{\mathrm{T}} \times i^{2}(s \mathrm{~A}) w^{\mathrm{T}}-\left(i(s \mathrm{~A}) w^{\mathrm{T}}\right)^{2}} \times \sqrt{w(s \mathrm{~A}) w^{\mathrm{T}} \times w(s \mathrm{~A})\left(i^{2}\right)^{\mathrm{T}}-\left(w(s \mathrm{~A})^{\mathrm{T}}\right)^{2}}} \\
& =\frac{s^{2}\left(w \mathrm{~A} w^{\mathrm{T}} \times i \mathrm{~A} i^{\mathrm{T}}\right)-s^{2}\left(i \mathrm{~A} w^{\mathrm{T}} \times w \mathrm{~A} i^{\mathrm{T}}\right)}{\sqrt{s^{2}\left(w \mathrm{~A} w^{\mathrm{T}} \times i^{2} \mathrm{~A} w^{\mathrm{T}}-\left(i \mathrm{~A} w^{\mathrm{T}}\right)^{2}\right)} \times \sqrt{s^{2}\left(w \mathrm{~A} w^{\mathrm{T}} \times w \mathrm{~A}\left(i^{2}\right)^{\mathrm{T}}-\left(w \mathrm{~A} i^{\mathrm{T}}\right)^{2}\right)}} \\
& =\frac{s^{2}\left(w \mathrm{~A} w^{\mathrm{T}} \times i \mathrm{~A} i^{\mathrm{T}}-i \mathrm{~A} w^{\mathrm{T}} \times w \mathrm{~A} i^{\mathrm{T}}\right)}{s^{2}\left(\sqrt{w \mathrm{~A} w^{\mathrm{T}} \times i^{2} \mathrm{~A} w^{\mathrm{T}}-\left(i \mathrm{~A} w^{\mathrm{T}}\right)^{2}} \times \sqrt{w \mathrm{~A} w^{\mathrm{T}} \times w \mathrm{~A}\left(i^{2}\right)^{\mathrm{T}}-\left(w \mathrm{~A} i^{\mathrm{T}}\right)^{2}}\right)} \\
& =\frac{w \mathrm{~A} w^{\mathrm{T}} \times i \mathrm{~A} i^{\mathrm{T}}-i \mathrm{~A} w^{\mathrm{T}} \times w \mathrm{~A} i^{\mathrm{T}}}{\sqrt{w \mathrm{~A} w^{\mathrm{T}} \times i^{2} \mathrm{~A} w^{\mathrm{T}}-\left(i \mathrm{~A} w^{\mathrm{T}}\right)^{2}} \times \sqrt{w \mathrm{~A} w^{\mathrm{T}} \times w \mathrm{~A}\left(i^{2}\right)^{\mathrm{T}}-\left(w \mathrm{~A} i^{\mathrm{T}}\right)^{2}}}
\end{aligned}
$$

Each of the terms expressed as matrix multiplications in equation (8) can also be equivalently expressed as a combination of sums, outer products $(\otimes)$ and Hadamard products $(\circ)$; the motivation for this will become clear below. Summarizing the equivalences: 


$$
\begin{aligned}
n & =w \mathrm{~A} w^{\mathrm{T}}=\sum_{r, c} \mathrm{~A}_{r, c} \\
\sum r_{i} & =i \mathrm{~A} w^{\mathrm{T}}=\sum_{r, c}(i \otimes w \circ \mathrm{A})_{r, c} \\
\sum c_{i} & =w \mathrm{~A} i^{\mathrm{T}}=\sum_{r, c}(w \otimes i \circ \mathrm{A})_{r, c} \\
\sum r_{i} c_{i} & =i \mathrm{~A} i^{\mathrm{T}}=\sum_{r, c}(i \otimes i \circ \mathrm{A})_{r, c} \\
\sum r_{i}^{2} & =i^{2} \mathrm{~A} w^{\mathrm{T}}=\sum_{r, c}\left(i^{2} \otimes w \circ \mathrm{A}\right)_{r, c} \\
\sum c_{i}^{2} & =w \mathrm{~A}\left(i^{2}\right)^{\mathrm{T}}=\sum_{r, c}\left(w \otimes i^{2} \circ \mathrm{A}\right)_{r, c}
\end{aligned}
$$

We can now express $\hat{\rho}$ as:

$$
\hat{\rho}=\frac{\sum_{r, c} \mathrm{~A}_{r, c} \times \sum_{r, c}(i \otimes i \circ \mathrm{A})_{r, c}-\sum_{r, c}(i \otimes w \circ \mathrm{A})_{r, c} \times \sum_{r, c}(w \otimes i \circ \mathrm{A})_{r, c}}{\sqrt{\sum_{r, c} \mathrm{~A}_{r, c} \times \sum_{r, c}\left(i^{2} \otimes w \circ \mathrm{A}\right)_{r, c}-\left(\sum_{r, c}(i \otimes w \circ \mathrm{A})_{r, c}\right)^{2}} \times \sqrt{\sum_{r, c} \mathrm{~A}_{r, c} \times \sum_{r, c}\left(w \otimes i^{2} \circ \mathrm{A}\right)_{r, c}-\left(\sum_{r, c}(w \otimes i \circ \mathrm{A})_{r, c}\right)^{2}}}
$$

Visually, these computations can be represented as Hadamard products of the matrix A with various weighting matrices reflecting combinations of the row and column indices (although it is more accurate to think of the matrix A itself serving as the weights, and the row and column indices are the "real" data points whose correlation is being computed):

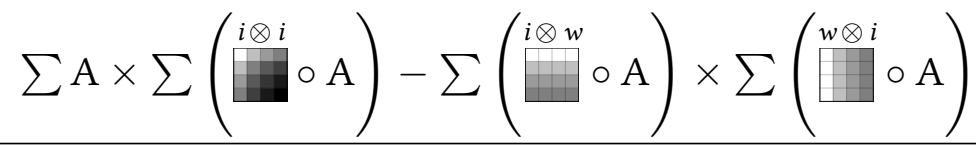

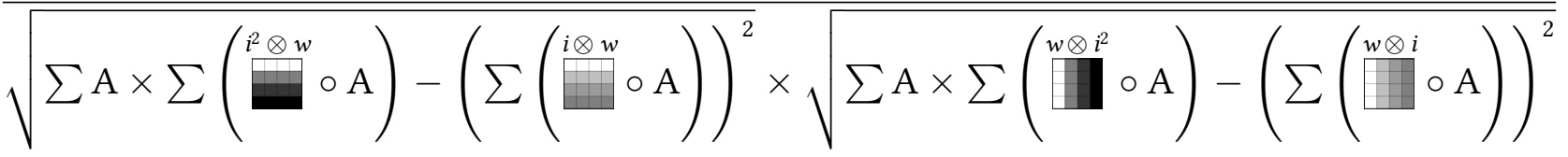

Example computations follow with different kinds of matrices substituted for A:

Diagonal matrix:

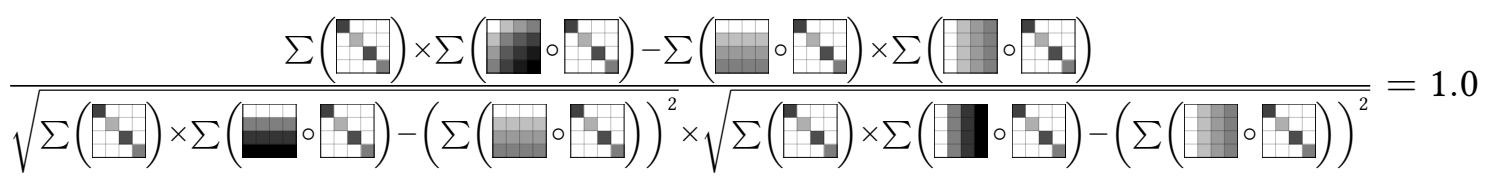


Tridiagonal matrix:

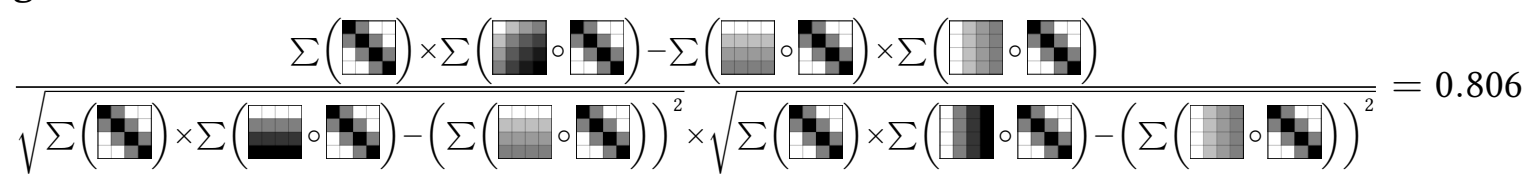

Uniform matrix:

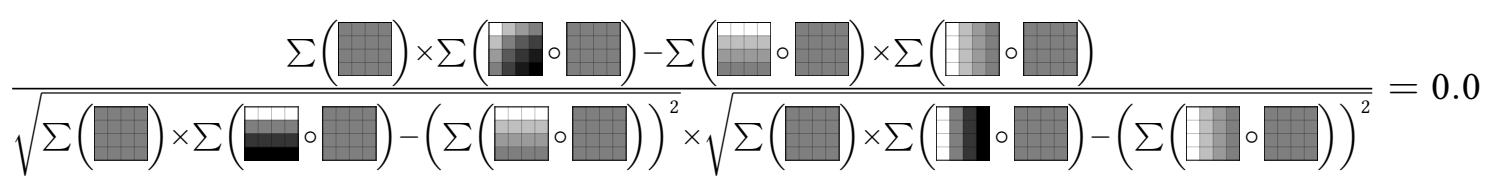

Random matrix:

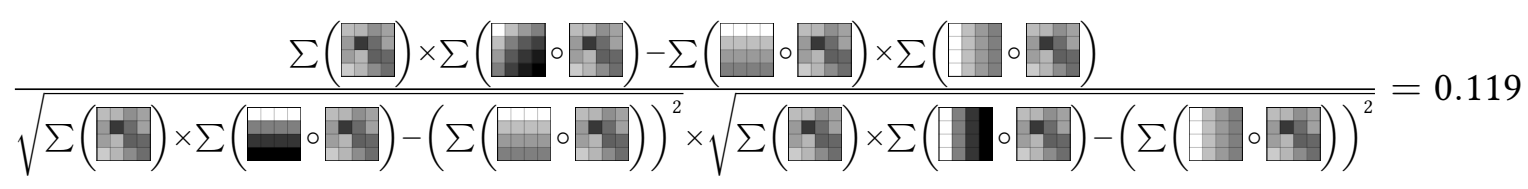

Minor diagonal matrix:

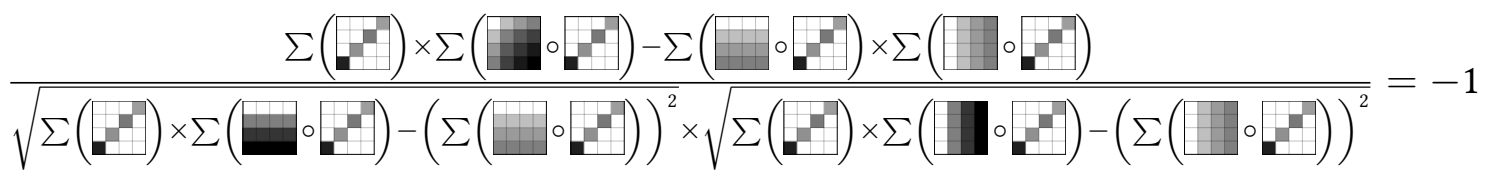

\section{DERIVATION OF THE SECOND FORM OF THE PEARSON EQUATION}

Repeating equation (2) here, but with more familiar $(x, y)$ coordinates in place of the $(c, r)$ notation used in equation (2):

$$
\hat{\rho}=\frac{\sum_{i=1}^{n}\left(x_{i}-\bar{x}\right)\left(y_{i}-\bar{y}\right)}{\sqrt{\sum_{i=1}^{n}\left(x_{i}-\bar{x}\right)^{2}} \times \sqrt{\sum_{i=1}^{n}\left(y_{i}-\bar{y}\right)^{2}}}
$$

We begin by examining only the numerator: 
original numerator

$$
\sum_{i=1}^{n}\left(x_{i}-\bar{x}\right)\left(y_{i}-\bar{y}\right)
$$

after multiplying the binomial terms $\sum_{i=1}^{n}\left(x_{i} y_{i}-x_{i} \bar{y}-\bar{x} y_{i}+\bar{x} \bar{y}\right)$

after distributing the summation

$$
\sum x_{i} y_{i}-\sum x_{i} \bar{y}-\bar{x} \sum y_{i}+\bar{x} \bar{y} n
$$

after rewriting $\bar{x}$ and $\bar{y}$

$$
\sum x_{i} y_{i}-\sum x_{i} \frac{\sum y_{i}}{n}-\frac{\sum x_{i}}{n} \sum y_{i}+\frac{\sum x_{i}}{n} \frac{\sum y_{i}}{n} n
$$

after canceling $n$ in final term

$$
\sum x_{i} y_{i}-\sum x_{i} \frac{\sum y_{i}}{n}-\frac{\sum x_{i}}{n} \sum y_{i}+\frac{\sum x_{i}}{n} \sum y_{i}
$$

after canceling last two terms

$$
\sum x_{i} y_{i}-\sum x_{i} \frac{\sum y_{i}}{n}
$$

after multiplying by $\frac{n}{n}$

$$
\frac{1}{n}\left(n \sum x_{i} y_{i}-\sum x_{i} \sum y_{i}\right)
$$

Since the contents of each squareroot in the denominator of equation (10) have the same form as the original numerator, the same sequence of steps will convert the standard deviation along the $\mathrm{x}$ dimension $\sqrt{\sum\left(x_{i}-\bar{x}\right)^{2}}$ into $\sqrt{\frac{1}{n}\left(\sum x_{i}^{2}-\left(\sum x_{i}\right)^{2}\right)}$, and likewise for the corresponding $y$ term, yielding:

$$
\hat{\rho}=\frac{\frac{1}{n}\left(n \sum x_{i} y_{i}-\sum x_{i} \sum y_{i}\right)}{\sqrt{\frac{1}{n}\left(n \sum x_{i}^{2}-\left(\sum x_{i}\right)^{2}\right)} \sqrt{\frac{1}{n}\left(n \sum y_{i}^{2}-\left(\sum y_{i}\right)^{2}\right)}}
$$

and after cancelling the $\frac{1}{n}$ terms, we have reached the form of equation (3):

$$
\hat{\rho}=\frac{n \sum x_{i} y_{i}-\sum x_{i} \sum y_{i}}{\sqrt{n \sum x_{i}^{2}-\left(\sum x_{i}\right)^{2}} \sqrt{n \sum y_{i}^{2}-\left(\sum y_{i}\right)^{2}}}
$$

\section{SUPPLEMENTARY FIGURES}

\section{Stimulus duration and alignment}

To reduce the influence of early cortical representations of acoustic-phonetic information, we performed analyses on both the full temporal span of each epoch and on truncated epochs (beginning at $100 \mathrm{~ms}$ after the consonant-vowel transition of the stimulus syllable). Figure S2 shows the alignment and duration of each stimulus, grouped by onset consonant. 


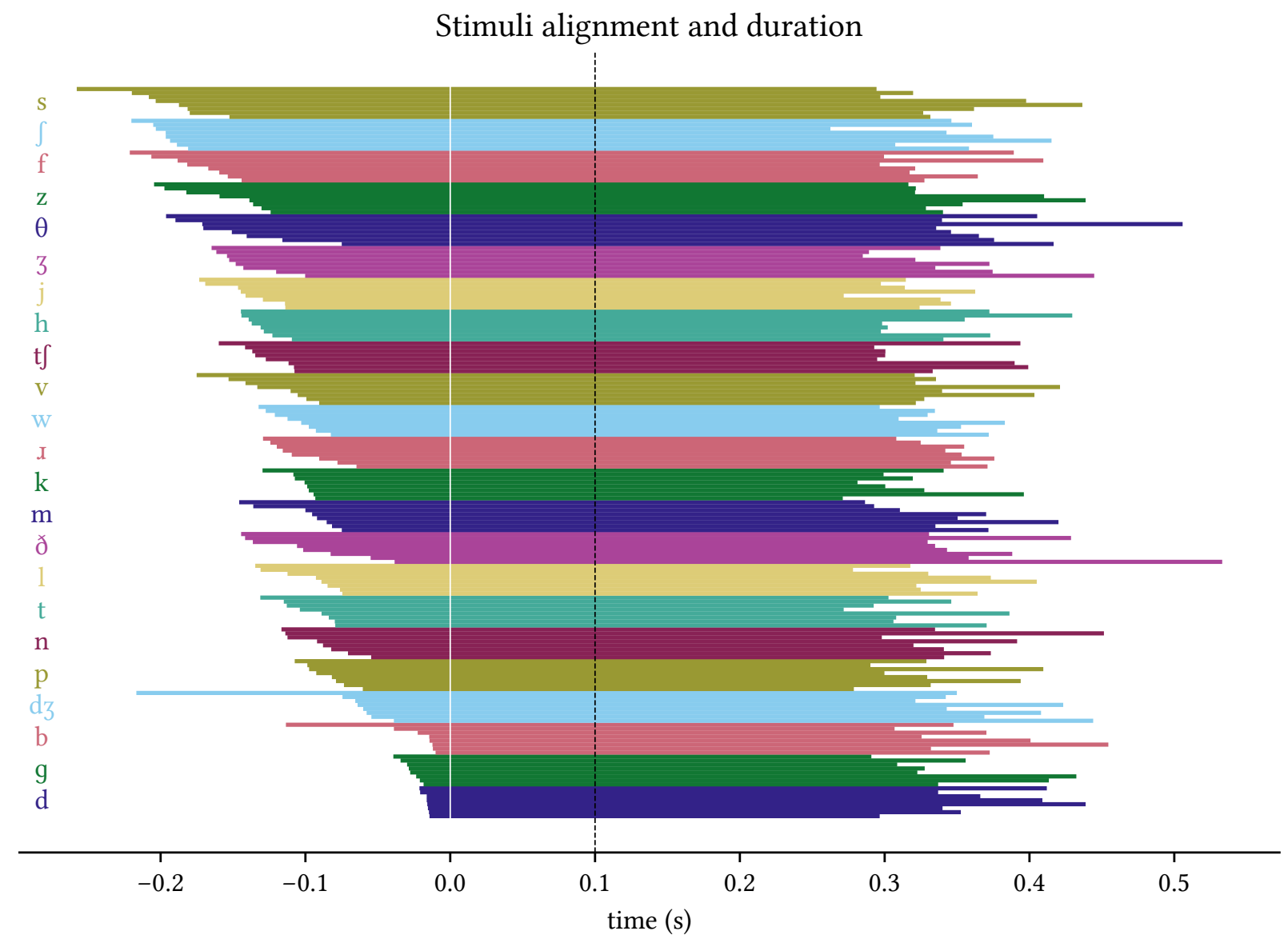

Figure S2: Durations and alignments of all English syllables used as stimuli. The vertical white line marks the consonant-vowel transition point, and the dashed black line indicates the truncation point for the truncated-epoch analysis.

\section{Signal-to-noise ratio}

One potential pitfall when analyzing neurophysiological data is variability in signal-to-noise ratio (SNR) across subjects (this can be especially problematic for group comparisons between patient and control populations). To ensure that SNR was not the driving factor in our measure of matrix diagonality, we performed some basic analyses of SNR in our data. Figure S3 shows the SNR, blink detection performance, and trial retention rate for each subject; figure $\mathrm{S} 4$ shows the (lack of) relationship between SNR and the computed "matrix diagonality" values for each subject in each phonological feature system. 
SNR: $10 \times \log _{10}($ evoked power / baseline power $)$
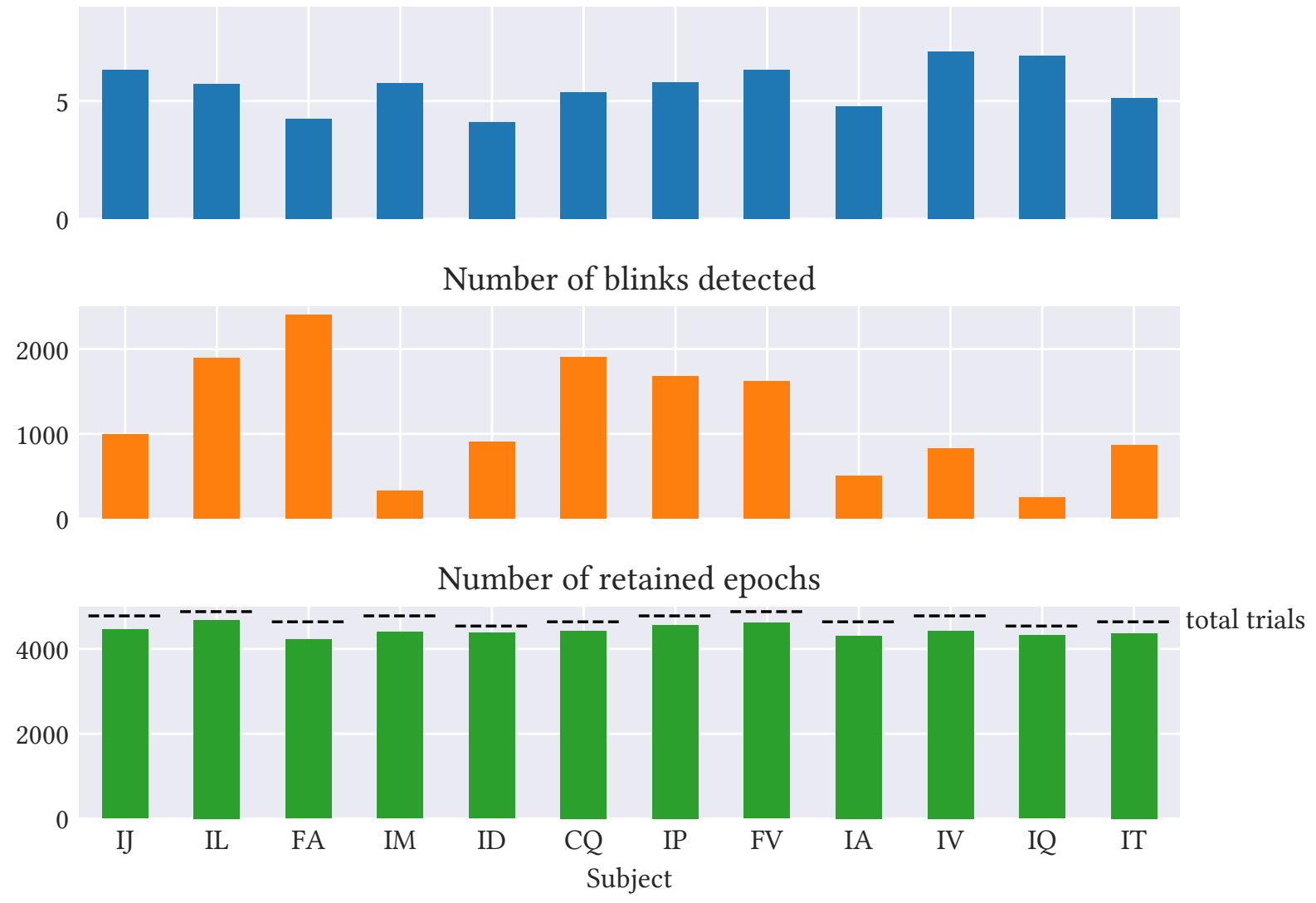

Figure S3: Plots of SNR, blink detection, and retained epochs for each subject. The total number of trials varies slightly for each subject due to additional (unanalyzed) trials from foreign talkers; each listener heard only 2 of the 4 foreign talkers, and the languages represented by the foreign talkers did not necessarily have equal numbers of consonants.

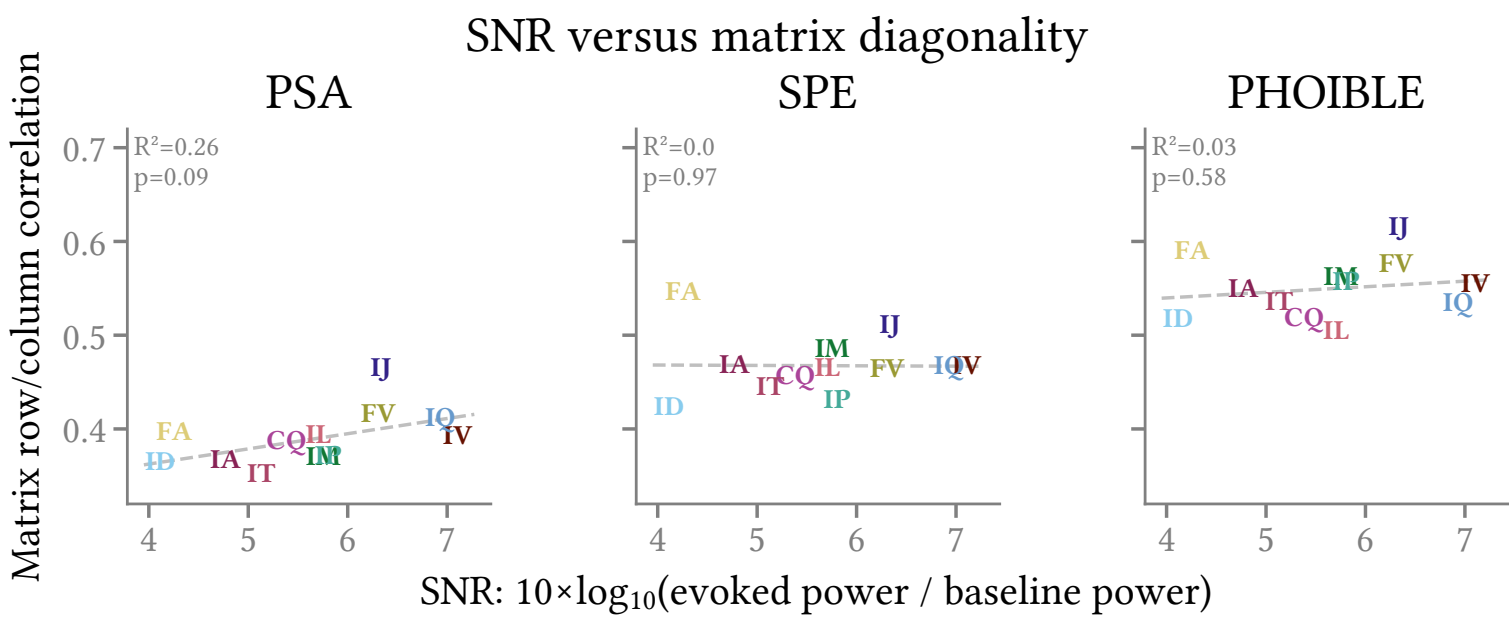

Figure S4: SNR versus matrix diagonality for each phonological feature system. Two-letter codes are unique subject identifiers. There is no evidence to support a correlation between SNR and matrix diagonality in any of the three feature systems (all p-values $>0.05$, uncorrected). 


\section{Accuracy of individual pairwise classifiers}

The mean accuracy across subjects for any given pairwise comparison was generally above $90 \%$. Figure S5 shows the accuracy of each individual pairwise comparison, along with the distribution of values for each subject.

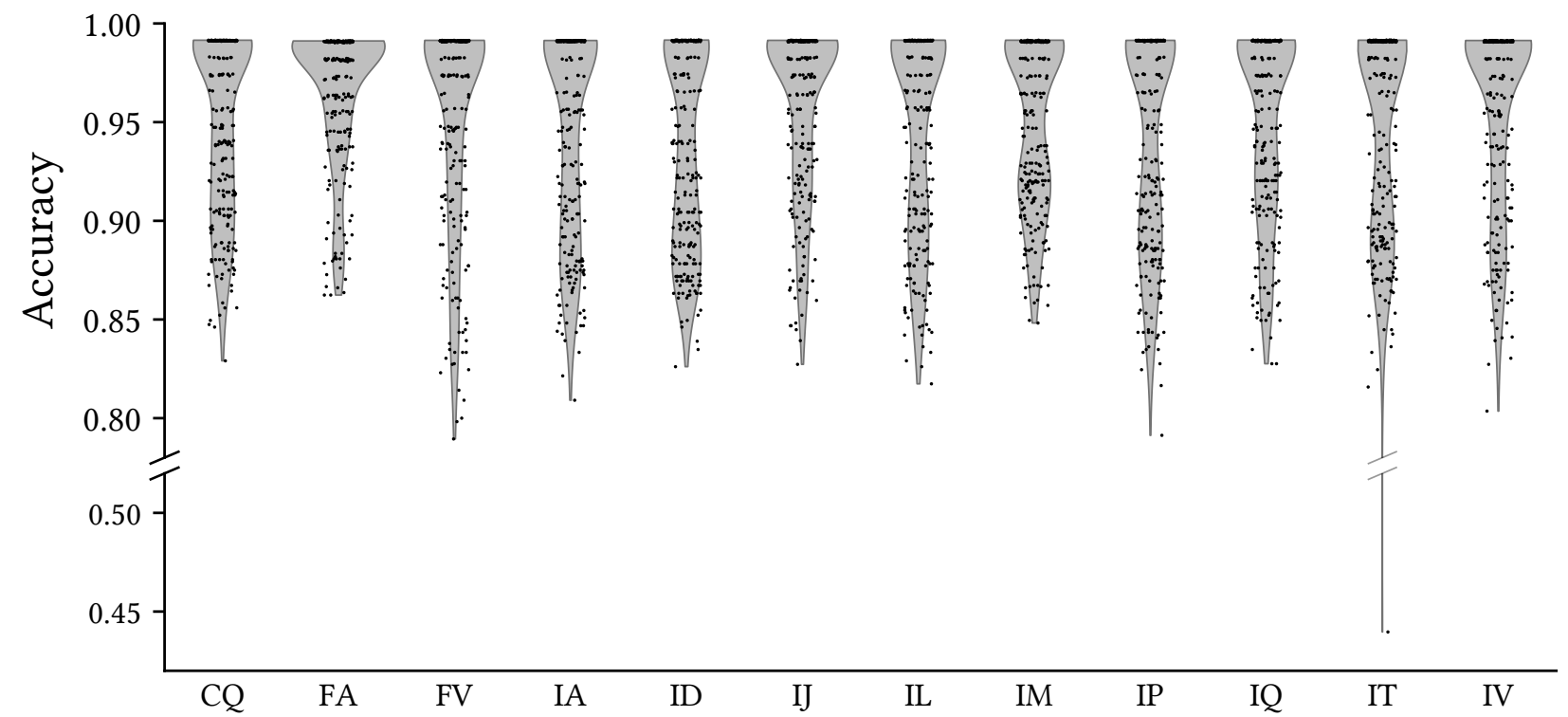

Figure S5: Violin plot of accuracy scores for pairwise classifiers, with overlaid strip plots (jittered along the horizontal dimension). The outlier point for subject IT is the pairwise classifier for $/ \mathrm{n} /$ versus $/ \mathrm{p} /$.

\section{Confusion matrices for individual subjects}

Here we present confusion matrices for each of the three phonological feature systems, computed separately for each subject. Cross-subject similarity is good; confusion matrices for two subjects for a given phonological feature system are generally more similar than confusion matrices for a single subject for two different phonological feature systems. 


\section{PSA feature-based classifiers' prediction scores by subject}

$0.001 \%$

$0.01 \%$

$0.1 \%$

$1 \%$

$10 \%$

$100 \%$

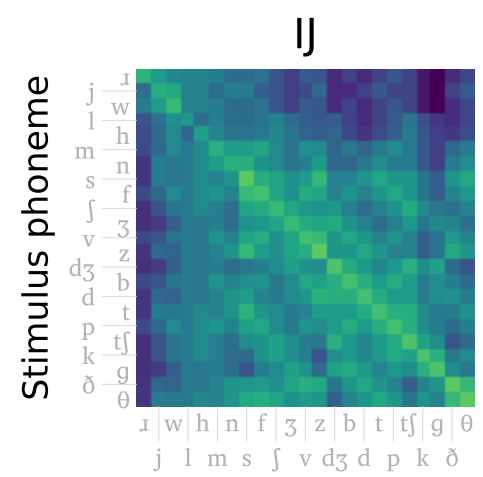

IL

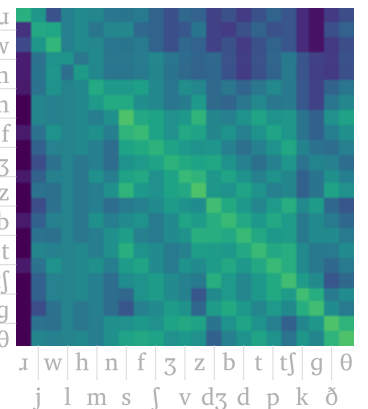

FA

IM

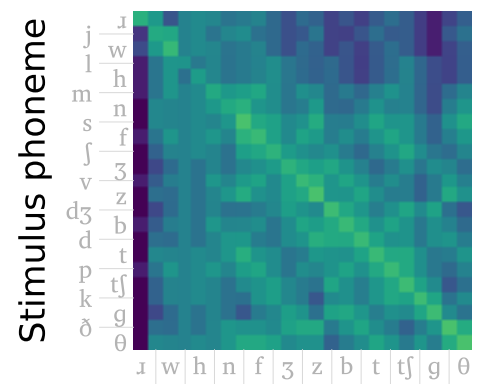

ID

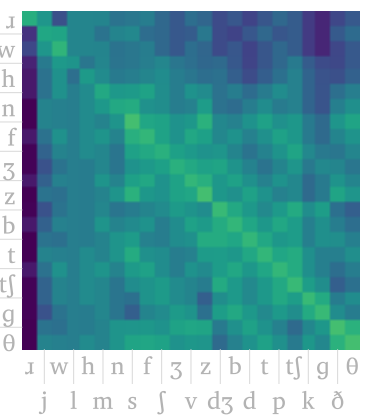

FV

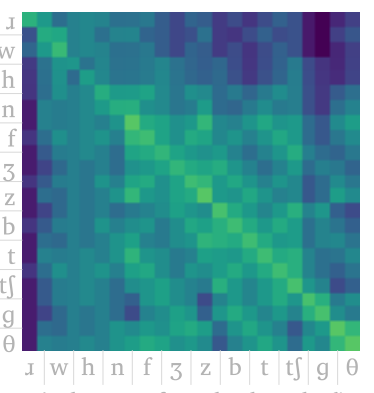

IQ

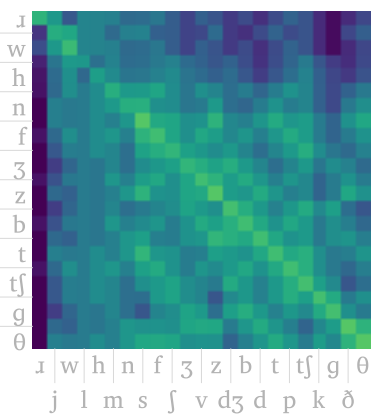

CQ

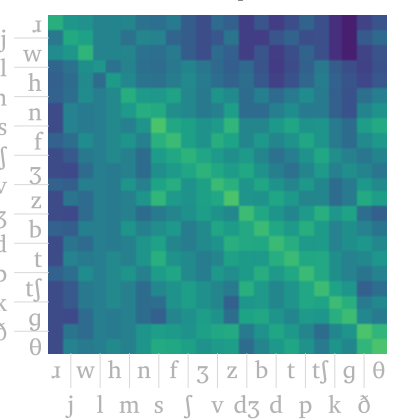

IA

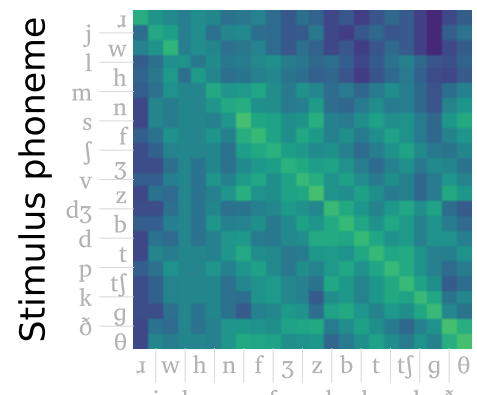

IV

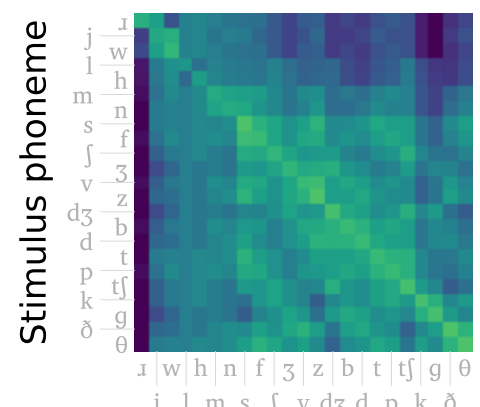

Predicted phoneme

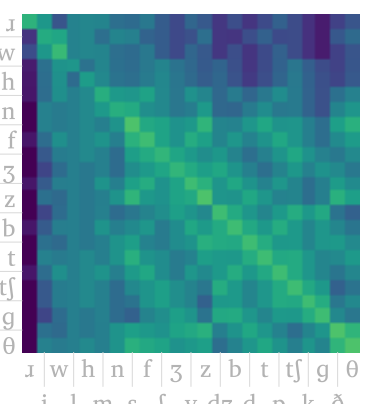

Predicted phoneme

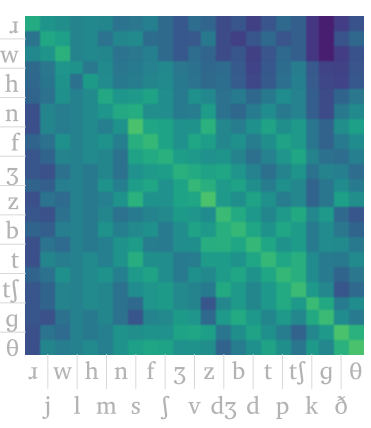

IT

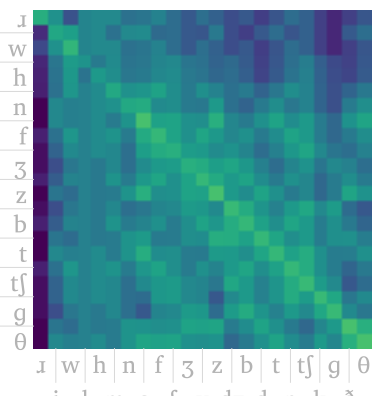

Predicted phoneme

Figure S6: Confusion matrices for each subject for the PSA phonological feature system classifiers. 


\section{SPE feature-based classifiers' prediction scores by subject}
$0.001 \%$
$0.01 \%$
$0.1 \%$
$1 \%$
$10 \%$
$100 \%$

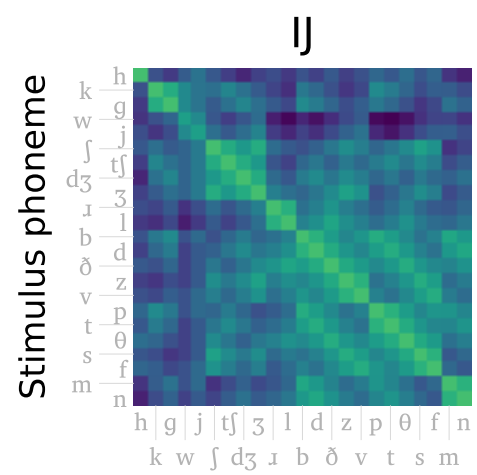

IM

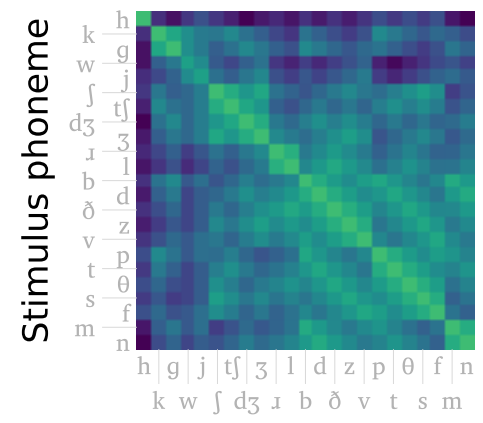

IP

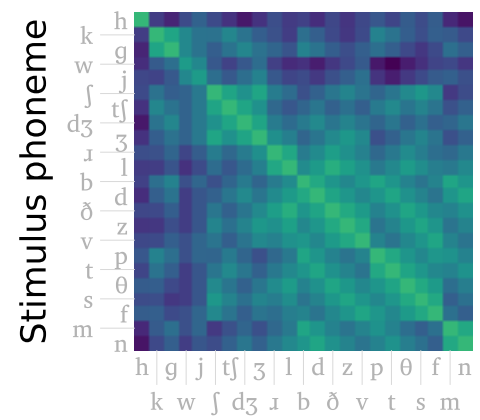

IV

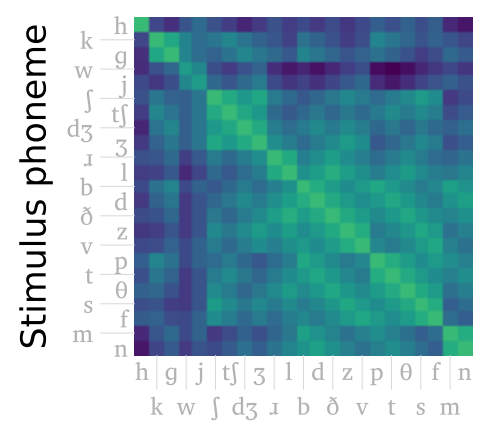

Predicted phoneme
IL

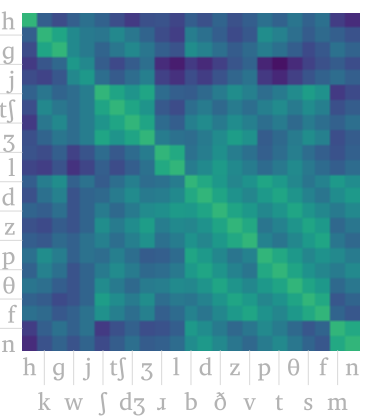

ID

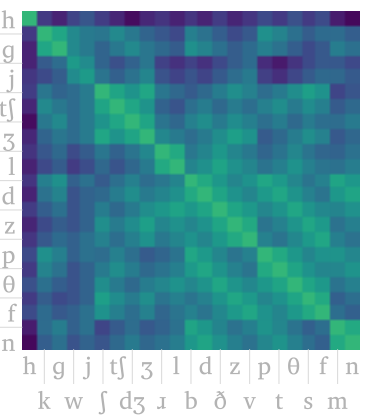

FV

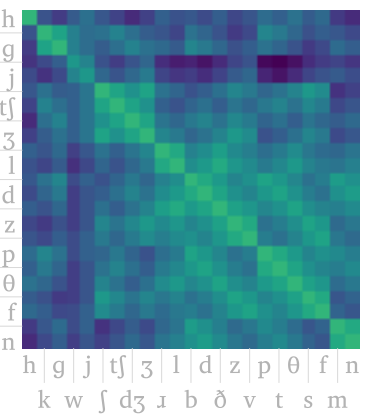

IQ

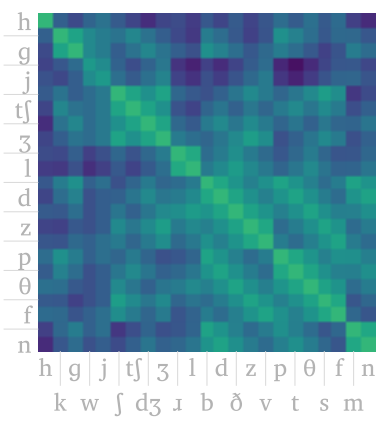

Predicted phoneme
FA

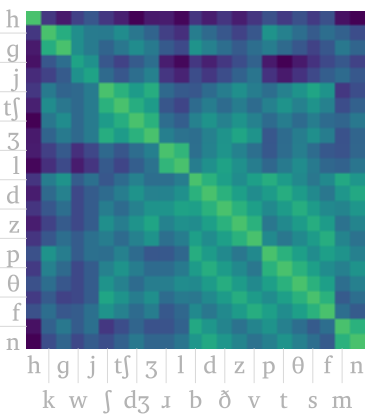

$\mathrm{CQ}$

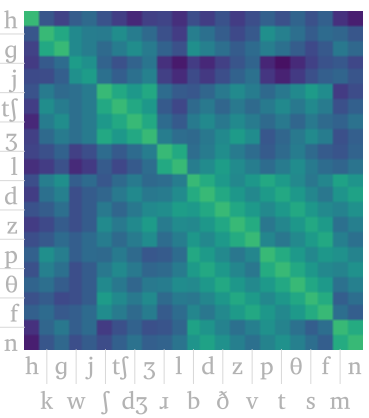

IA

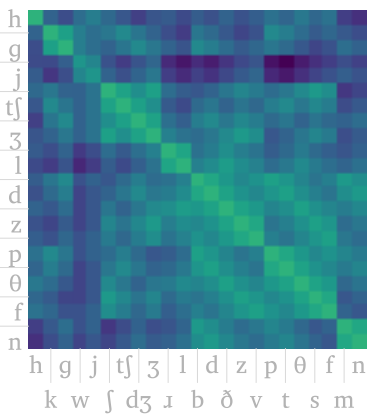

IT

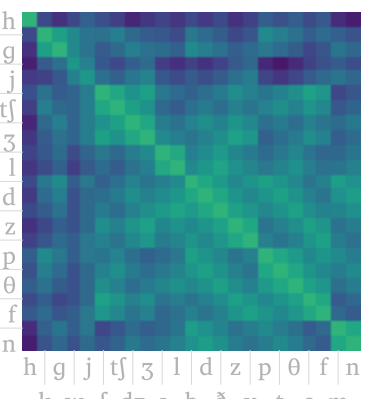

Predicted phoneme

Figure S7: Confusion matrices for each subject for the SPE phonological feature system classifiers. 


\section{PHOIBLE feature-based classifiers' prediction scores by subject}
$0.001 \%$
$0.01 \%$
$0.1 \%$
$1 \%$
$10 \%$
$100 \%$

IJ

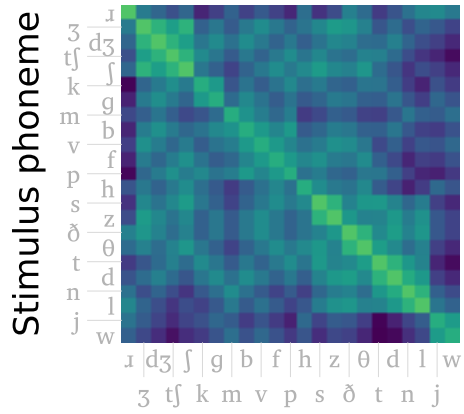

IM

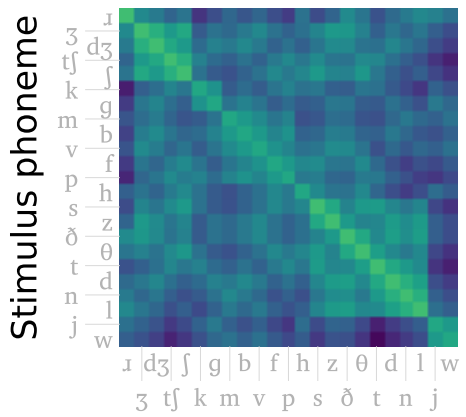

IP

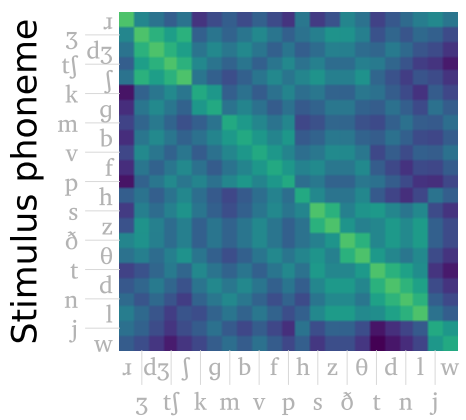

IV

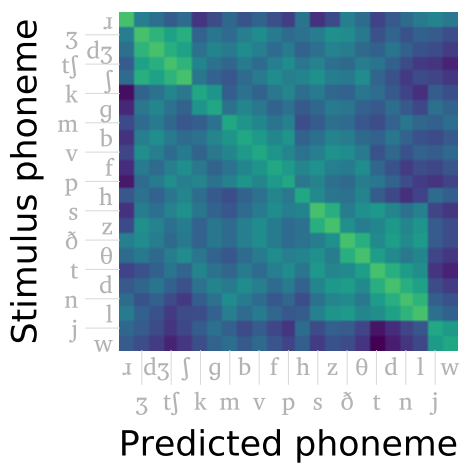

IL

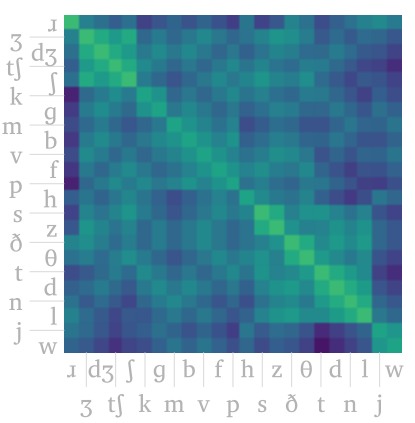

ID

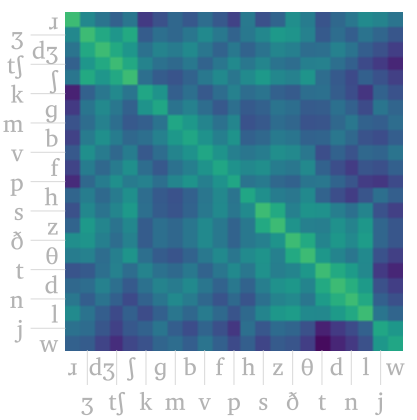

FV

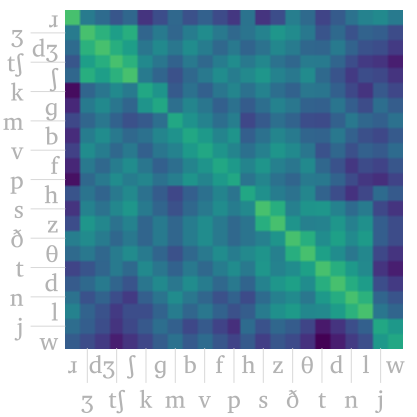

IQ

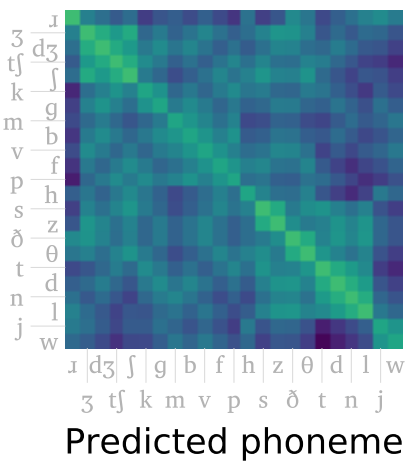

FA

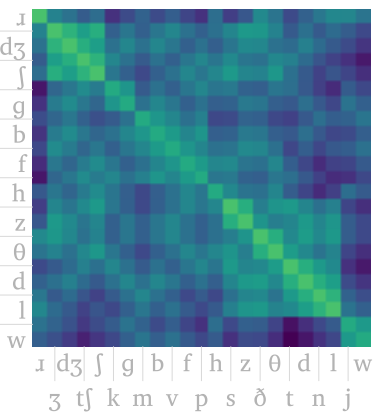

CQ

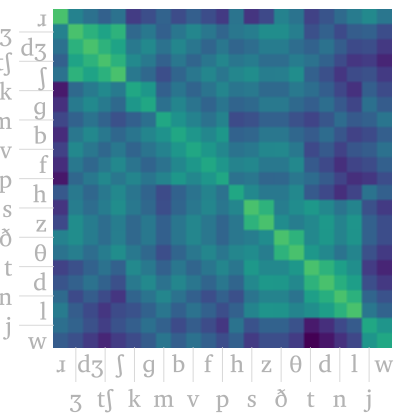

IA

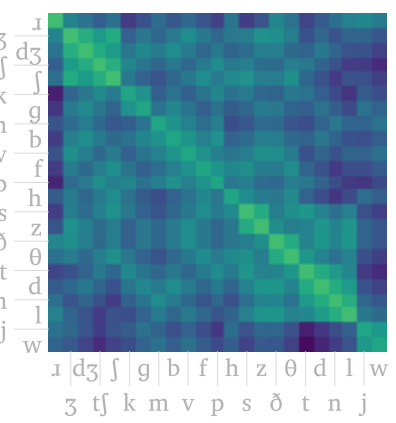

IT

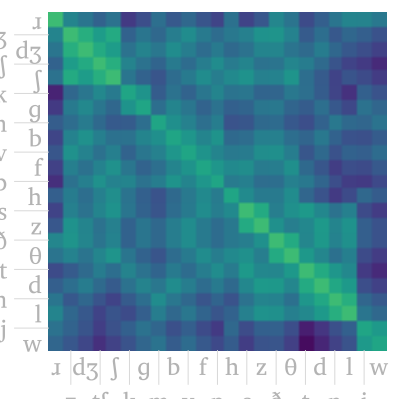

Predicted phoneme

Figure S8: Confusion matrices for each subject for the PHOIBLE phonological feature system classifiers. 


\section{fointly-ordered confusion matrices}

Here we present confusion matrices for each of the three phonological feature systems, with row order determined by hierarchical clustering of the rows performed jointly across the three matrices. This allows direct comparison of particular rows or submatrices across the three plots.
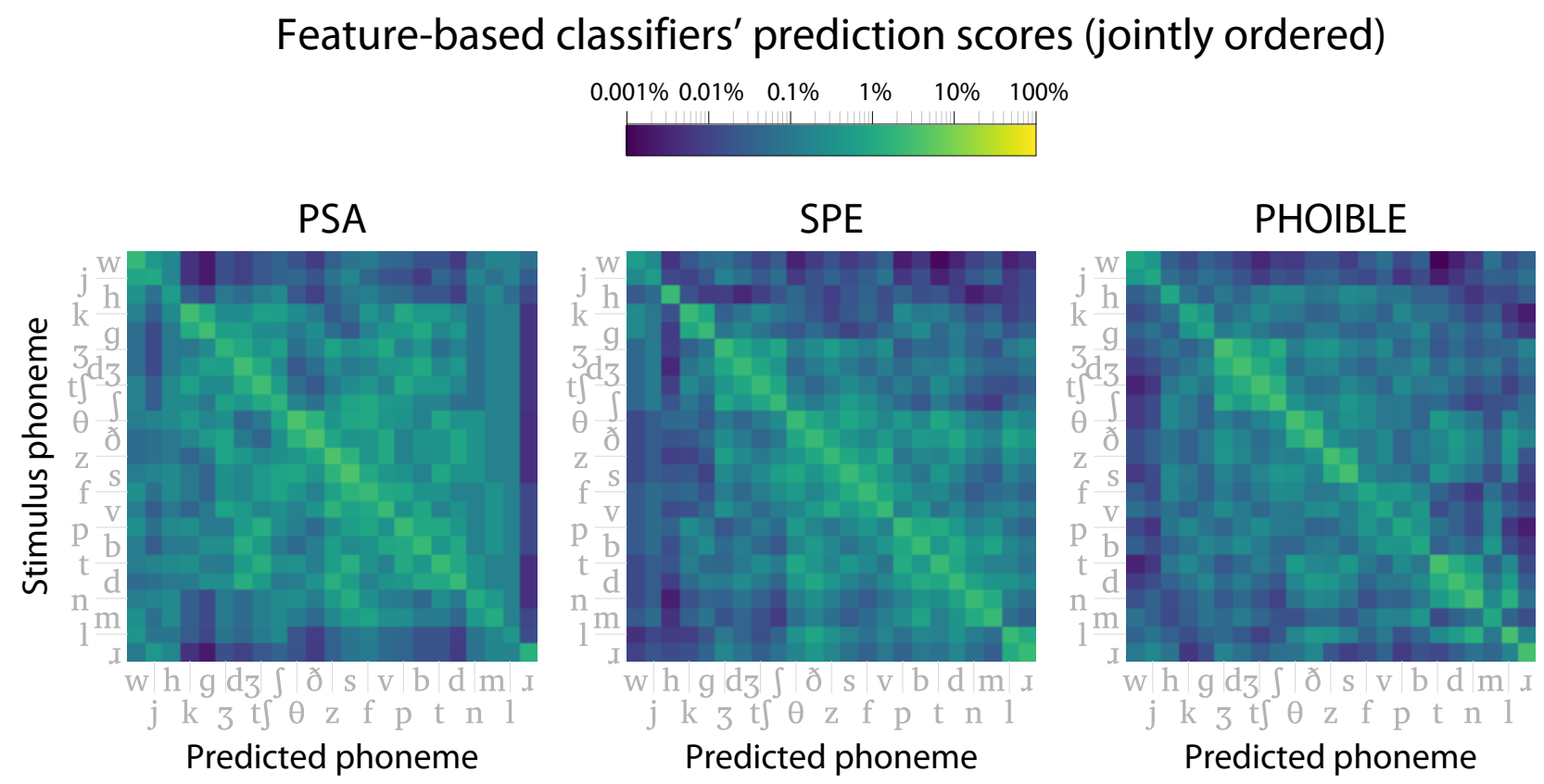

Figure S9: Confusion matrices for each feature system, with consistent row and column order across all three matrices.

\section{REFERENCES}

Tad. (2015). Measure of "how much diagonal" a matrix is (Version 2015-08-12). Mathematics Stack Exchange. Retrieved from https://math.stackexchange.com/a/1393907 